\title{
Design and characterization of specMACS, a multipurpose hyperspectral cloud and sky imager
}

\author{
Florian Ewald ${ }^{1,3, *}$, Tobias Kölling ${ }^{1, *}$, Andreas Baumgartner ${ }^{2}$, Tobias Zinner ${ }^{1}$, and Bernhard Mayer ${ }^{1}$ \\ ${ }^{1}$ Ludwig Maximilians Universität, Institut für Meteorologie, Munich, Germany \\ ${ }^{2}$ Deutsches Zentrum für Luft und Raumfahrt, Institut für Methodik der Fernerkundung, Oberpfaffenhofen, Germany \\ ${ }^{3}$ Deutsches Zentrum für Luft und Raumfahrt, Institut für Physik der Atmosphäre, Oberpfaffenhofen, Germany \\ *These authors contributed equally to this work.
}

Correspondence to: Tobias Zinner (tobias.zinner@lmu.de)

Received: 7 August 2015 - Published in Atmos. Meas. Tech. Discuss.: 22 September 2015

Revised: 27 March 2016 - Accepted: 31 March 2016 - Published: 4 May 2016

\begin{abstract}
The new spectrometer of the Munich Aerosol Cloud Scanner (specMACS) is a multipurpose hyperspectral cloud and sky imager designated, but is not limited to investigations of cloud-aerosol interactions in Earth's atmosphere. With its high spectral and spatial resolution, the instrument is designed to measure solar radiation in the visible and shortwave infrared region that is reflected from, or transmitted through clouds and aerosol layers. It is based on two hyperspectral cameras that measure in the solar spectral range between 400 and $2500 \mathrm{~nm}$ with a spectral bandwidth between 2.5 and $12.0 \mathrm{~nm}$. The instrument was operated in ground-based campaigns as well as aboard the German High Altitude LOng Range (HALO) research aircraft, e.g., during the ACRIDICON-CHUVA campaign in Brazil during summer 2014.

This paper describes the specMACS instrument hardware and software design and characterizes the instrument performance. During the laboratory characterization of the instrument, the radiometric response as well as the spatial and spectral resolution was assessed. Since the instrument is primarily intended for retrievals of atmospheric quantities by inversion of radiative models using measured radiances, a focus is placed on the determination of its radiometric response. Radiometric characterization was possible for both spectrometers, with an absolute accuracy of $3 \%$ at their respective central wavelength regions. First measurements are presented which demonstrate the wide applicability of the instrument. They show that key demands are met regarding the radiometric and spectral accuracy which is required for the intended remote sensing techniques.
\end{abstract}

\section{Introduction}

The spectrally resolved measurement of solar radiation is a long-standing method in earth science. In the first half of the twentieth century, Gordon Dobson introduced the method of spectroscopy into the field of atmospheric remote sensing. Since then, the exploitation of atmospheric and particle absorption has led to the development of spaceborne instruments like the Moderate-resolution Imaging Spectroradiometer (MODIS) for the remote sensing of cloud properties or the Infrared Atmospheric Sounding Interferometer (IASI) to retrieve trace gas profiles.

Remote sensing of cloud and aerosol parameters is still mostly done with multispectral sensors, i.e., using only a limited number of spectral channels. Prominent examples are, e.g., ground-based aerosol retrievals using CIMEL sun photometers in the Atmospheric Radiation Measurement Program and the Aerosol Robotic Network (AERONET; Holben et al., 1998) or satellite-based multichannel techniques following Hansen and Pollack (1970); Twomey and Cocks (1989) and Nakajima and King (1990) for remote sensing of cloud properties. However, the application of spectrally resolved, hyperspectral techniques in cloud and aerosol remote sensing is still in its early stages.

First steps towards hyperspectral techniques for cloud remote sensing were done with instruments like the Solar Spectral Flux Radiometer (SSFR, Pilewskie et al., 2003) or the Spectral Modular Airborne Radiation measurement sysTem (SMART, Wendisch et al., 2001; Wendisch and Mayer, 2003) from a ground-based (McBride et al., 2011; Chiu et al., 2012; 
Jäkel et al., 2013) or an airborne perspective (Ehrlich et al., 2008; Eichler et al., 2009; Schmidt et al., 2007; Coddington et al., 2010). All of these methods are based on non-imaging sensors; i.e., only one measurement is taken at a time and one line of measurements is constructed by sensor motion or cloud motion over a ground-based measurement.

There are some imaging spectroscopy instruments for the ground-based or airborne remote sensing perspective. In the visible wavelength range, one of the earliest instruments was the Compact Airborne Spectrographic Imager (CASI, Babey and Anger, 1989) with 288 spectral channels (2.5 nm resolution). Further cloud remote sensing applications were done with the AisaEAGLE instrument from SPECIM, which covers the spectral range between 400 and $970 \mathrm{~nm}$ with a spectral resolution of $2.9 \mathrm{~nm}$ (Bierwirth et al., 2013; Schäfer et al., 2015). The Airborne Visible/InfraRed Imaging Spectrometer (AVIRIS, Green et. al., 1998) extended the measurement range into the near-infrared spectrum with 224 spectral channels ( $10 \mathrm{~nm}$ resolution) between 400 and $2500 \mathrm{~nm}$. A further imaging spectroscopy instrument is the Airborne Prism EXperiment (APEX) imaging spectrometer (Itten et al., 2008; Schaepman et al., 2015), with 532 spectral channels and a spectral resolution between 0.9 and $12.3 \mathrm{~nm}$.

As commercially available spectral imagers for measurements in the solar visible and near-infrared spectrum are becoming more and more affordable they are more frequently being used nowadays. For airborne remote sensing of land surfaces a few, still costly commercial solutions are available at the moment. Based on spectral off-the-shelf camera systems, the Meteorological Institute of the University of $\mathrm{Mu}$ nich decided to tailor a system to its specific needs. In the following this new hyperspectral imaging instrument for atmospheric measurements on ground-based and airborne platforms with a spectral coverage of $400-2500 \mathrm{~nm}$ will be introduced and characterized in detail. Based on some first applications, the scientific data obtained with the specMACS instrument will be introduced.

\subsection{Conceptual embedding}

The institute hosts a range of instruments for remote measurements of the atmosphere: an aerosol lidar, a millimeterwave cloud radar, a sun photometer, and multiple differential optical absorption spectroscopy (DOAS) instruments. Using this active and passive instrumentation, improvements of our understanding of the aerosol-cloud interaction in the atmosphere are pursued. Cloud microphysical development like droplet growth, glaciation processes, and ice nucleation as well as cloud dynamics, is influenced by the abundance and type of available cloud nuclei, the fraction of the aerosol background which can act as a nucleus for droplet or ice particle growth. These microphysical processes are of greatest interest for the understanding of future climate development (Houghton et al., 2001).
To this end, Marshak et al. (2006a); Martins et al. (2011); Zinner et al. (2008) and Ewald et al. (2013) proposed cloud side scanning measurements to observe the basically vertical development of cloud particles. To retrieve particle size and thermodynamic phase they propose to use reflected solar radiation in the near-visible to near-infrared spectral regions. This application is a core goal for the development of the new sensor. With the help of an imaging spectrometer, the required spatially resolved measurements become possible. Especially the vertical dimension of these observations should reflect many aspects of cloud-aerosol interaction as well as mixing of cloudy and ambient air (Martins et al., 2011; Rosenfeld et al., 2012). For the same partially cloudy scenes, additional remote measurements of interacting aerosol characteristics (particle type, size, amount) as well as of some gaseous atmospheric components will become accessible by exploitation of the spectral image information.

\subsection{Accuracy considerations}

The complexity of cloud geometry and three-dimensional (3D) radiative effects related to it pose a great challenge to cloud side remote sensing. Various studies of Varnai and Marshak (2002); Marshak et al. (2006b) and Zinner and Mayer (2006) quantified the impact of 3-D radiative effects on particle size retrievals based on 1-D radiation transfer simulations like Nakajima and King (1990), and found an overestimation of effective radius by up to $2 \mu \mathrm{m}$ (Cornet et al., 2005) with a standard deviation of $1.5 \mu \mathrm{m}$ (Bréon and Doutriaux-Boucher, 2005). Especially for spatially high resolved cloud side measurements $(<100 \mathrm{~m})$ standard deviations to the true effective radius can be $20 \%$ or more (Zinner and Mayer, 2006).

The proposed retrieval method by Martins et al. (2011) and Zinner et al. (2008) tries to account for this uncertainty by means of a statistical retrieval based on fully 3-D radiative transfer simulations. For optically thick liquid water clouds, an uncertainty in effective radius of $2 \mu \mathrm{m}$ relates roughly to a radiance uncertainty of $20 \%$ at the near-infrared wavelength $2100 \mathrm{~nm}$ used in the retrieval of Nakajima and King (1990). To limit the uncertainty in microphysical retrievals due to sensor characteristics, we aim for an absolute radiometric calibration uncertainty well below the retrieval uncertainty.

Spectral accuracy requirements are not too strict for current microphysical cloud retrievals, as no specific molecular absorption features are evaluated. However, the solar spectrum itself exhibits many narrow absorption bands. For this reason, the spectral accuracy should be comparable or better than the spectral bandwidth of the instrument. The radiometric accuracy can be compromised if resolved absorption bands are spectrally misaligned. Furthermore, measurements of accurate and highly resolved spectra are invaluable for the application of novel retrieval techniques, since various spectral atmospheric and soil features become exploitable. High 
spectral resolution measurements are needed in the visible near-infrared (VNIR) spectral range where many narrow absorption features are located, e.g., for photon path analysis using the optical depth of the oxygen A-band or for the detection of surface albedo influence based on known spectral vegetation features. Conceivable use of the spectral data to estimate the oxygen A-band depth tightens spectral accuracy requirements to a few nanometers or less (Fischer and Grassl, 1991). As shown by Heidinger and Stephens (2000), the retrieval of the total column optical depth of the oxygen A-band is limited by the spectral resolution of the instrument.

This work is based on previous work which developed hyperspectral instruments and their calibration. The general principle of measurement and the specific implementation of the hyperspectral instrument used was developed and described in detail by Aikio (2001). Jørgensen (2002) examined this design and described necessary steps in its calibration, potential error sources, and their mitigation. The overall approach to the calibration is based on the work of Lenhard et al. (2015) and was carried out in close cooperation with the Remote Sensing Technology Institute (IMF) of the German Aerospace Center (DLR).

This paper is organized as follows: Sect. 2 first introduces the new specMACS instrument and its measurement principle. Next, all necessary technical amendments and software developments are introduced that make specMACS a versatile and accurate cloud remote sensing instrument usable for airborne push-broom applications as well as for groundbased cloud side or hemispheric scans. In Sect. 3 the methods used during characterization and calibration of the instrument are introduced and described. Following each subsection, detailed results of the radiometric and spectral sensor characterization are given and discussed. Finally, application examples are shown, presenting the first airborne deployment of the instrument on-board HALO, the German high-altitude long-range research aircraft (Sect. 4). Cloud side measurements were collected through a customized side window of the aircraft during the Brazilian-German ACRIDICONCHUVA campaign in autumn 2014.

\section{The specMACS instrument}

The spectrometer of the Munich Aerosol Cloud Scanner (specMACS) is an imaging spectrometer system for the measurement of solar radiation in the $400-2500 \mathrm{~nm}$ wavelength range, which is based on two hyperspectral cameras. It is designed for remote sensing of cloud and aerosol optical properties and atmospheric trace gases. The emphasis is on the development of new ground-based retrieval methods of clouds as well as on the understanding of 3-D radiative effects in existing retrieval methods. Key properties of the two imaging spectrometers that were determined in this work are given in Table 1. The instrument was developed at the Me-
Table 1. Properties of the two SPECIM imaging spectrometers employed in specMACS for so-called visible, near-infrared, and shortwave infrared spectral ranges as characterized in this work. Here, FOV means the field of view of the complete spatial line, while IFOV denotes the instantaneous field of view of single pixels, which determines the spatial resolution along- and across-track.

\begin{tabular}{lll}
\hline & VNIR & SWIR \\
\hline Detector & SiO2 CMOS & HgCdTe CMOS \\
Spectral range & $417-1016 \mathrm{~nm}$ & $1015-2496 \mathrm{~nm}$ \\
Spectral bandwidth & typ. $2.5-4 \mathrm{~nm}$ & typ. $7.5-12 \mathrm{~nm}$ \\
FOV & $32.7^{\circ}$ & $35.5^{\circ}$ \\
IFOV (across-track) & typ. $1.4 \mathrm{mrad}$ & typ. $3.8 \mathrm{mrad}$ \\
IFOV (along-track) & typ. $2.0 \mathrm{mrad}$ & typ. $1.8 \mathrm{mrad}$ \\
Spatial pixels & 1312 & 320 \\
Spectral channels & 800 & 256 \\
Radiometric quantization & $12 \mathrm{bit}$ & $14 \mathrm{bit}$ \\
Usable dynamic range & $9.5 \mathrm{bit}$ & typ. $11-11.6 \mathrm{bit}$ \\
Max. frame rate & $145 \mathrm{~Hz}$ & $103 \mathrm{~Hz}$ \\
Temp. control & uncooled & $200 \mathrm{~K}$ \\
\hline
\end{tabular}

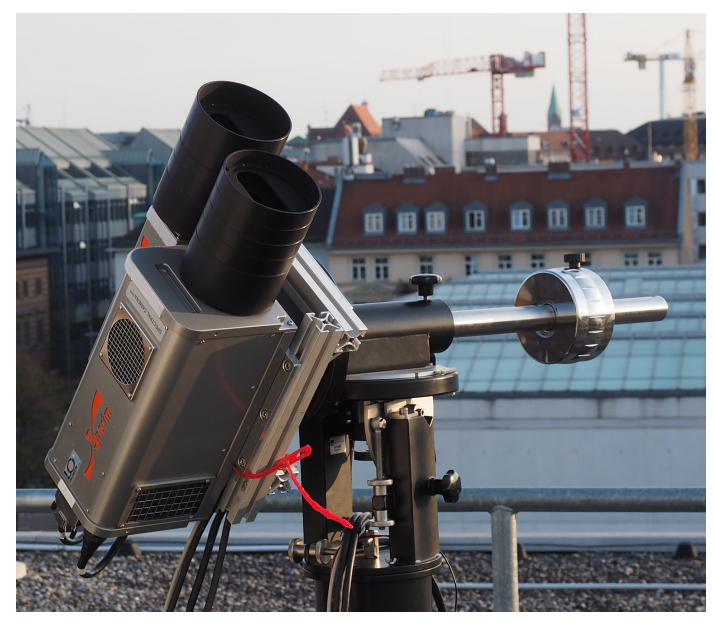

Figure 1. specMACS VNIR and SWIR sensors on the scanning mount. The stray light protection is prominently visible in front of the sensors.

teorological Institute of the Ludwig Maximilian University and is usually operated on the roof platform of the institute.

\subsection{Instrument concept}

The instrument comprises two commercially available hyperspectral line spectrometers built by SPECIM (Specim Spectral Imaging Ltd., Oulu, Finland.). Combined, these hyperspectral cameras simultaneously acquire light spectra between 400 and $2500 \mathrm{~nm}$ for one spatial dimension. The measurement principle is based on the diffraction of a light beam by a volume-phase holographic transmission grating after one spatial dimension has been filtered by an entrance slit $(\approx 30 \mu \mathrm{m}$ for both spectrometers $)$ as shown in Fig. 2. Af- 


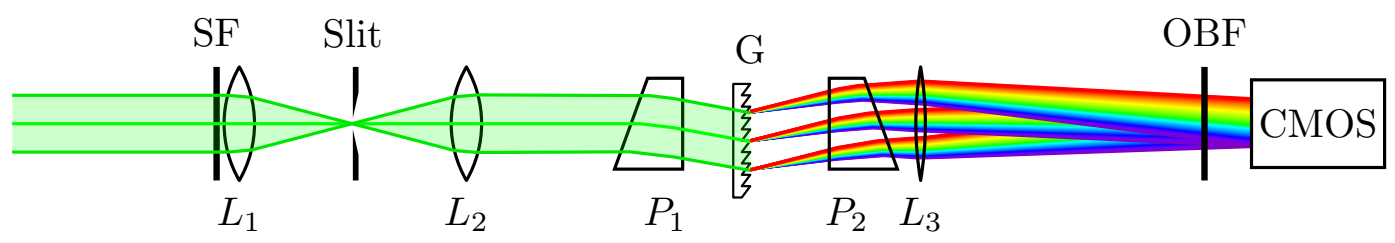

Figure 2. Optical concept of hyperspectral imagers. Sketch of the light path entering the instrument as it first gets spatially filtered by a slit and subsequently separated by a holographic grating: SF denotes the spectral flattening filter, $L_{1}$ the front optics, $L_{2}$ the collimator, $P_{1}$ the entry prism, $\mathrm{G}$ the volume-phase holographic transmission grating, $P_{2}$ the exit prism, $L_{3}$ the focuser, OBF the order blocking filter, CMOS the imaging sensor.

ter the grating, the spatial variations of the radiant flux are captured on one dimension of a complementary metal-oxide semiconductor (CMOS) active pixel sensor (APS; Fossum, 1997), while the spectral variations are registered on the other dimension. An order blocking filter (OBF) is mounted just in front of the APS to prevent spectral overlap of different diffraction orders. A detailed description of the measurement principle and the specific implementation of the used hyperspectral instrument can be found in Aikio (2001).

\subsection{VNIR spectrometer}

The spectral camera PFD (SPECIM SP-PFD-CL-65-V10E) is used for the coverage of the visible and near-infrared wavelength range $(400-1000 \mathrm{~nm}$; which in the following is referred to as the visible near-infrared, VNIR). It is equipped with a $18.5 \mathrm{~mm} f / 2.4$ front lens (OLE18.5). Inside the spectrograph (ImSpector V10E) the entrance slit, the collimating optics, the prism-grating-prism element, and the focusing optics are firmly connected together. Its linear dispersion is specified with $97.5 \mathrm{~nm} \mathrm{~mm}^{-1}$. In front of the sensor region corresponding to longer wavelengths originating from firstorder $m=-1$ diffraction, an order blocking filter (SPECIM OBF 570) is placed to prevent light of shorter wavelengths from second-order $m=-2$ diffraction reaching the sensor. The sensor is based on a camera (MV1-D1312-160) from Photonfocus, which is built around the monochrome, uncooled CMOS active pixel sensor (A1312). This sensor is backside-illuminated to increase its low-light performance. It provides a resolution of $1312 \times 800$ pixels with a pixel distance on chip (pixel pitch) of $8 \mu \mathrm{m} \times 8 \mu \mathrm{m}$ and an active optical area of $10.48 \mathrm{~mm} \times 8.64 \mathrm{~mm}$. The field of view (FOV) along the spatial line is $32.7^{\circ}$, while the instantaneous field of view (IFOV) for a single pixel is $1.37 \mathrm{mrad}$ across and $2.00 \mathrm{mrad}$ along the spatial line. The entrance slit width of $30 \mu \mathrm{m}$ limits the average spectral resolution to $3.1 \mathrm{~nm}$ with an average spectral sampling of $0.8 \mathrm{~nm}$. Due to noise, the usable dynamic range for a single frame of the VNIR camera is approximately 9.5 bit. Further parameters can be found in Table 1.

\subsection{SWIR spectrometer}

For the wavelength region between 1000 and $2500 \mathrm{~nm}$, the SWIR spectrometer (SPECIM SP-SWIR-LVDS-100-N25E) is used (which in the following is referred to as the shortwave infrared, SWIR). It is equipped with a $15 \mathrm{~mm} f / 2.1$ front optic lens (OLES15). Since the solar radiance decreases strongly from 1000 to $2500 \mathrm{~nm}$, the usable dynamic range over the complete wavelength range would be very limited. A spectral flattening filter (Hebo RC 01, SPECIM) is therefore placed in front of the lens to attenuate the shorter wavelengths and thereby improve the overall use of the dynamic range of the sensor for solar radiation. This filter has an additional special coating to block the wavelength range from 800 to $960 \mathrm{~nm}$, since the SWIR sensor is sensitive from $800 \mathrm{~nm}$ onwards and since these wavelengths cannot be filtered by an order blocking filter. The linear dispersion of its spectrograph (ImSpector N25E) is specified with $208 \mathrm{~nm} \mathrm{~mm}^{-1}$. Similar to the VNIR, an order blocking filter (OBF 1400) is placed in front of the SWIR sensor to prevent spectral overlap from different diffraction orders. The SWIR spectrometer uses the MARS SW $320 \times 256$ sensor from SOFRADIR with a pixel pitch of $30 \mu \mathrm{m}$. The HgCdTe-based detector is thermoelectrically cooled to $200 \mathrm{~K}$ to reduce the level of dark current noise. The FOV along the spatial line is $35.5^{\circ}$ while the IFOV is $3.79 \mathrm{mrad}$ across the spatial line and $1.82 \mathrm{mrad}$ along the spatial line. The entrance slit width of $30 \mu \mathrm{m} \mathrm{lim}-$ its the average spectral resolution to $10.3 \mathrm{~nm}$ with an average spectral sampling of $6.8 \mathrm{~nm}$. Due to the strongly varying dark signal, the noise-limited usable dynamic range for a single frame of the SWIR camera varies in the range of 11-11.6 bit, depending on integration time and environment conditions. More detailed information is given in Sect. 3.1.1. Further parameters are listed in Table 1.

\subsection{Stray light protection}

Both the VNIR and the SWIR sensors are affected by stray light, however, the effects on the SWIR sensor are typically a few times larger than on the VNIR. In Fig. 3, the effect of stray light and its mitigation is shown using a prototype of the actual stray light protector. To mitigate the effects of stray light permanently, a system of shielding baffles was designed 

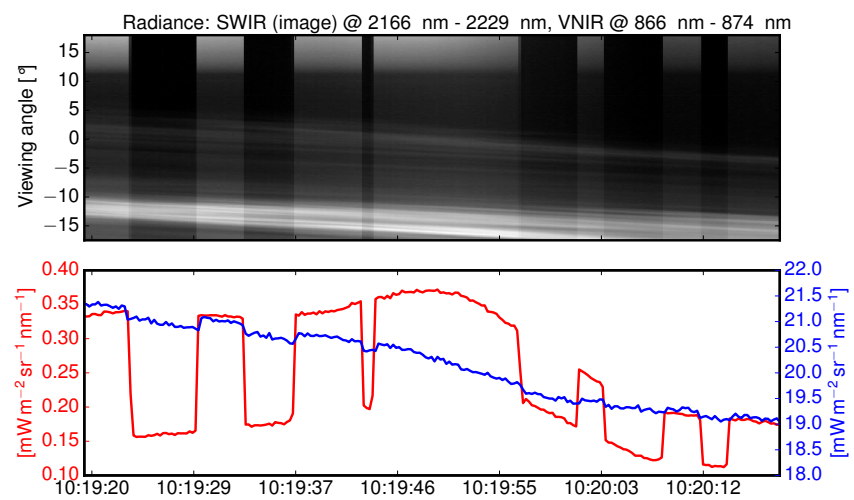

Figure 3. The top image shows a zenith pointing scene as captured with the SWIR camera. During the capture, stray light has been repeatedly shielded from the sensors with a prototype of the now permanently installed stray light protection. Due to the faint cloud cover, the radiance from inside the nominal field of view is very small and the stray light effect becomes very obvious. The lower plot shows the radiance averaged over the full field of view of the SWIR and VNIR sensors in red and blue respectively. On both sensors, a stray light effect and its mitigation is visible, however the effect on the VNIR is much less pronounced. This can be understood due to the brighter background illumination as well as due to less reflective fore optics of the sensor.

which is placed in front of the camera optics (Fig. 4). The stray light protector was built of aluminium which was sandblasted, anodized, and painted with NEXTEL velvet black paint. According to Dury et al. (2007), the regular and diffuse single scattering reflectance of this coating is typically less than $5 \%$ in the whole spectral range of both spectrometers. Simulations showed that these baffles attenuate incident light that originates from angles more than $15^{\circ}$ outside the FOV by at least 2-3 orders of magnitude. After implementing the stray light protection, no visible evidence of stray light was found during ground measurements with the sun outside the FOV by more than $15^{\circ}$.

\subsection{Instrument automation}

The specMACS hyperspectral imager is a multipurpose measurement system, which produces data at a rate of up to $300 \mathrm{MiB} \mathrm{s}^{-1}$ and which must be stored reliably on disk. Furthermore, the system must be autonomous. This applies in particular to an airborne deployment with limited operator availability.

During ground-based scans or unsupervised airborne operation, the integration time $t_{\text {int }}$ has to be adjusted to avoid over- as well as underexposure. Furthermore, frequent dark frame measurements are necessary since the dark current signal of the SWIR sensor varies strongly with integration time and ambient air temperature. For these reasons, a piece of control software automatically sets integration time and controls dark frame measurements, potentially resulting in

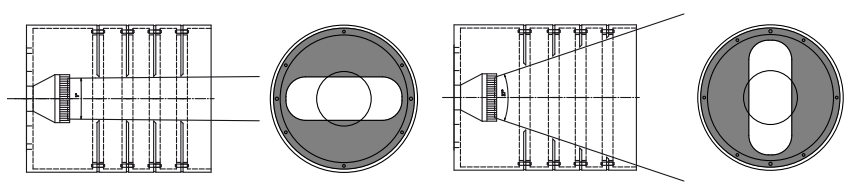

Figure 4. Design of stray light protection. Left: cut along spectral axis, right: cut along the spatial axis. The baffles have a length of $160 \mathrm{~mm}$ and a diagonal of $125 \mathrm{~mm}$.

additional (non-instantaneous) 5.6 bit of dynamic range for each sensor. Details of the automated control software are described in detail in the Appendix Sect. A.

In order to control and repeat complex measurement tasks, the CAMPaign ASsistant (CAMPAS) was developed. It is a domain-specific scripting language combined with a communication library which has access to all system components. Using CAMPAS, the user can define measurement tasks like a hemispherical scan through step-by-step instructions. These instructions control the behavior of the sensors, the scanning mount, and all other system components combined. The CAMPAS system furthermore provides simple access to various dynamic coordinates (e.g., sun position or the current position of other instruments). Thus, a general task can be written once and repeated many times which increases comparability of results.

\section{Characterization and calibration methods}

There are three essential characteristics which define the overall performance of imaging spectrometers. First, the radiometric response of the instrument has to be known to obtain absolute radiometric measurements. Secondly, a precise knowledge of the spectral projection onto the sensor is required for a calibrated pixel to wavelength relationship. To conclude, information about the spatial projection and its geometric distortions is required to assess the spatial image quality and its resolution. In contrast to the stable sensor characteristics, faster-varying data, like sensor settings, orientation, and dark signal need to be captured during measurements. By using this information, measured raw data can be converted into physical units during the calibration procedure.

A guideline through the whole process and the involved quantities is given by the calibration flow chart in Fig. 5. The process follows Lenhard et al. (2015) closely and is extended by a nonlinearity correction regarding integration time explained in Sect. 3.1.2. The following subsections will cover each of the displayed steps.

In the following, all variables are given as pixel-wise properties when not mentioned otherwise. Temporal averaged properties of the sensor will be identified with angle brackets while spatial averages will be indicated with an overbar. The laboratory characterization of the specMACS sensors 


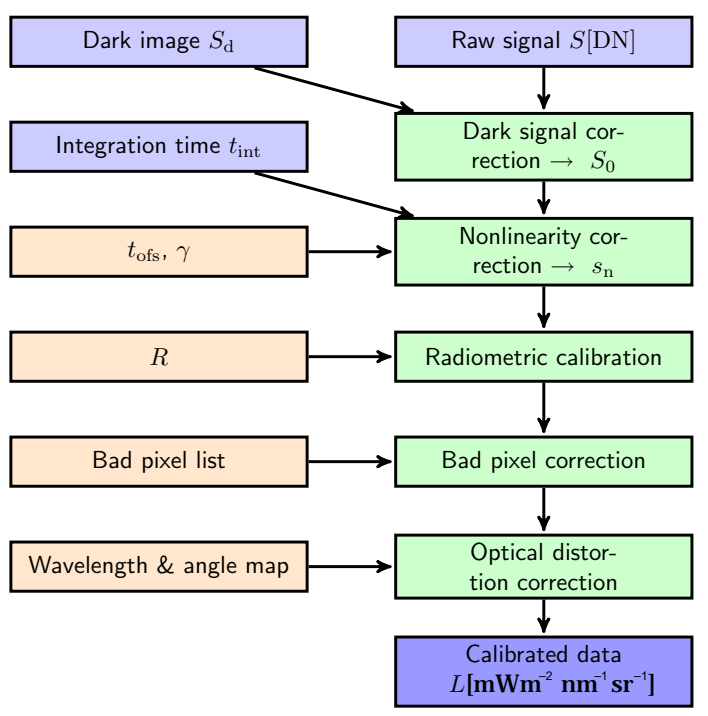

Figure 5. Schematic calibration using the newly developed nonlinearity correction. Light blue boxes are measured data, orange boxes are characterization data, green boxes are calibration steps, and dark blue is the calibrated data.

was performed at the Calibration Home Base (CHB; Gege et al., 2009) of the Remote Sensing Technology Institute of the German Aerospace Center.

\subsection{Radiometric characterization}

The sensors consist of independent pixels, where each acts as a radiance sensor for its specific spectral and spatial section of the full image. For this reason, pixel sensors are subject to inter-pixel variations caused by imperfections in the sensor material and electronics. These variations are almost constant in time but become evident on uncorrected images as a visible noise pattern. This pattern is generally called fixed pattern noise (FPN) and can be mitigated through calibration using characterized parameters determined in the following sections. Long-term variations of the FPN can be covered through periodic recharacterization of the sensor.

Each pixel outputs the measured signal as a digital number (DN). To obtain an absolute radiometric value the sensor has to be calibrated since its signal is subject to influences other than the impinging light. The sensor signal $S$ can be modeled as a sum of a radiometric signal $S_{0}$ containing only radiance information, $S_{\mathrm{d}}$, which describes the dark signal of the sensor and the noise $\mathcal{N}$ of the sensor:

$S=S_{0}+S_{\mathrm{d}}+\mathcal{N}$.

In the following subsections, dark signal $S_{\mathrm{d}}$, radiometric signal $S_{0}$, and noise $\mathcal{N}$ will be independently examined. In the remaining subsections, optical performance like angular and spectral bandwidth as well as keystone effects will be discussed.

\subsubsection{Dark signal}

Inherent to all electronic imaging sensors is the dark signal $S_{\mathrm{d}}$. It is a pixel-dependent offset and its variation between pixels is often described as dark signal nonuniformity. The total signal $S$ is composed of the photoelectric signal $S_{0}$, a dark signal $S_{\mathrm{d}}$, and the remaining noise $\mathcal{N}$ (Eq. 1). When the shutter is closed and the photoelectric signal $S_{0}$ becomes zero by definition, an averaged dark frame $\left\langle S_{\mathrm{d}}\right\rangle$ with very small remaining noise (as $\langle\mathcal{N}\rangle \rightarrow 0$ ) can directly be measured:

$\langle S\rangle=\left\langle S_{0}+S_{\mathrm{d}}+\mathcal{N}\right\rangle=\left\langle S_{\mathrm{d}}\right\rangle+\langle\mathcal{N}\rangle \approx\left\langle S_{\mathrm{d}}\right\rangle$.

The dark signal $S_{\mathrm{d}}$ is further composed of the dark current signal $S_{\mathrm{dc}}=s_{\mathrm{dc}} t_{\text {int }}$ and a read-out offset $S_{\text {read }}$ :

$S_{\mathrm{d}}=s_{\mathrm{dc}} t_{\text {int }}+S_{\text {read }}$.

The dark current $s_{\mathrm{dc}}$ originates from thermally generated electrons and holes within the semiconductor material. Since the electrons are randomly generated over time, the dark current signal $S_{\mathrm{dc}}$ increases linearly with $s_{\mathrm{dc}}$ and integration time $t_{\text {int }}$. The remaining offset $S_{\text {read }}$ is caused by the read-out process and is therefore independent of $t_{\text {int }}$.

The dark signal $S_{\mathrm{d}}\left(t_{0}\right)$ of an illuminated frame at time $t_{0}$ is estimated through linear interpolation of averaged dark frames $\left\langle S_{\mathrm{d}}\left(t_{-1}\right)\right\rangle$ and $\left\langle S_{\mathrm{d}}\left(t_{1}\right)\right\rangle$ measured at $t_{-1}$ before and $t_{1}$ after the image frame:

$$
\begin{aligned}
\left\langle S_{\mathrm{d}}^{*}\left(t_{0}\right)\right\rangle & =(1-w)\left\langle S_{\mathrm{d}}\left(t_{-1}\right)\right\rangle+w\left\langle S_{\mathrm{d}}\left(t_{1}\right)\right\rangle, \\
\text { with } w & =\frac{t_{0}-t_{-1}}{t_{1}-t_{-1}} .
\end{aligned}
$$

The photoelectric signal $S_{0}\left(t_{0}\right)$ (including the remaining noise $\mathcal{N})$ can then be estimated using the interpolated dark frame $\left\langle S_{\mathrm{d}}^{*}\left(t_{0}\right)\right\rangle$ :

$S_{0}\left(t_{0}\right)+\mathcal{N}=S\left(t_{0}\right)-S_{\mathrm{d}}\left(t_{0}\right) \approx S\left(t_{0}\right)-\left\langle S_{\mathrm{d}}^{*}\left(t_{0}\right)\right\rangle$.

Hereby, the linear interpolation leads to a dark signal uncertainty:

$\sigma_{\mathrm{d}}\left(t_{0}\right)=\sqrt{\sigma_{\mathrm{d}}^{2}\left(t_{0-}\right)(1-w)+\sigma_{\mathrm{d}}^{2}\left(t_{0+}\right) w}$.

This uncertainty results from standard deviations $\sigma_{\mathrm{d}}\left(t_{+1}\right)$ and $\sigma_{\mathrm{d}}\left(t_{-1}\right)$ of the individual dark signal averages at $t_{-1}$ and $t_{1}$ in combination with an upper estimate of the dark signal drift $\Delta \overline{S_{\mathrm{d}}}$ projected forward from $t_{-1}$ and backward from $t_{1}$ to time $t_{0}$ :

$2 \sigma_{\mathrm{d}}^{2}\left(t_{0-}\right)=2 \sigma_{\mathrm{d}}^{2}\left(t_{-1}\right)+\left(\Delta \overline{S_{\mathrm{d}}}\left(t_{0}-t_{-1}\right)\right)^{2}$
$2 \sigma_{\mathrm{d}}^{2}\left(t_{0+}\right)=2 \sigma_{\mathrm{d}}^{2}\left(t_{1}\right)+\left(\Delta \overline{S_{\mathrm{d}}}\left(t_{1}-t_{0}\right)\right)^{2}$.

To specify this uncertainty for actual measurements, the following analysis will investigate the dark signal characteristics, e.g., the maximal dark signal drift, of both sensors. 
The dark signal analysis was done under controlled laboratory conditions during calibration within the CHB facility as well as on one flight during the ACRIDICON-CHUVA campaign. In order to suppress the noise during lab analysis, 500 consecutive dark frames were averaged. Dark frames were measured for nine different integration times while ambient air temperatures were held constant by air conditioning. During the measurements, the temperature in the VNIR casing remained stable at $312.0 \mathrm{~K}$. Since the SWIR camera is not equipped with a temperature sensor, the VNIR temperature has been used as a proxy.

For the analysis during the flight, only 30 consecutive dark frames were averaged to minimize gaps between radiometric measurements. Analysis of mean dark signal levels $\overline{S_{\mathrm{d}}}$ in flight over all pixels was done for both spectrometers using integration times $t_{\text {int }}$ of 5,8 , and $12 \mathrm{~ms}$. During the $6 \mathrm{~h}$ flight, the ambient air temperature was gradually changing due to the cabin air-conditioning system. This led to fluctuating VNIR casing temperatures between 312.4 and $320.6 \mathrm{~K}$.

The analysis of the dark signal shows clear differences between the sensors used for the VNIR and the SWIR spectrometer. Figure 6 (right) shows mean dark signal levels $\overline{S_{\mathrm{d}}}$ in available digital number range when averaging over 30 dark frames as measured during a flight of the aircraft campaign ACRIDICON-CHUVA. The green curve shows the readings from a temperature sensor located within the casing of the VNIR spectrometer. While the level of $\overline{S_{\mathrm{d}}}$ for the SWIR depends on integration time $t_{\text {int }}$ as well as on temperature, the level of $\overline{S_{\mathrm{d}}}$ for the VNIR is independent of $t_{\mathrm{int}}$ and temperature. Maximal dark current drifts $\Delta \overline{S_{\mathrm{d}}}$ of $30 \mathrm{DN} / \mathrm{min}$ were found for the SWIR, while $\Delta \overline{S_{\mathrm{d}}}$ remained below $6 \mathrm{DN} / \mathrm{min}$ for the VNIR. In the following, these values will be used as an upper estimate in the calculation of the $2 \sigma$ error of the dark signal in Eq. (6).

A more in-depth analysis of the dark signal behavior is shown in Fig. 6 (left), where measurements of $S_{\mathrm{d}}$ in a controlled lab environment (CHB) are compared with measurements of $S_{\mathrm{d}}$ during the flight. The slope of the regression line reflects the dependence of $\overline{S_{\mathrm{d}}}$ on $t_{\text {int }}$, while the shaded area shows the influence of temperature on this relationship. In Fig. 6 (left) the nonuniformity of $S_{\mathrm{d}}$ is shown by error bars, which is only significant for the SWIR. With temperature increasing from 310.4 to $320.0 \mathrm{~K}$, the number of thermally generated electrons per second increases from 352 to $494.0 \mathrm{DN} \mathrm{ms}^{-1}$ for the SWIR sensor. In this temperature range, the VNIR sensor shows only a marginal influence on dark signal levels. In contrast, a considerable amount of dynamic range is lost to the dark signal for the SWIR.

For the VNIR, there is mainly a fixed dark signal offset independent of $t_{\text {int }}$. Since thermally generated electrons do not play an important role in dark signal generation within the VNIR sensor the independence of $S_{\mathrm{d}}$ from $t_{\text {int }}$ and temperature is evident. In order to push the dark signal below the photoelectric signal, the HgCdTe-based detector of the SWIR spectrometer has to be cooled down to $200 \mathrm{~K}$. Although the
Table 2. Integration times (ms) used for nonlinearity measurements with the large integrating sphere.

\begin{tabular}{rrrrrr}
\hline \multirow{2}{*}{ VNIR } & 1.0 & 2.0 & 4.0 & 6.0 & 8.0 \\
& 10.0 & 12.0 & 14.0 & 16.0 & \\
\hline \multirow{2}{*}{ SWIR } & 0.1 & 0.3 & 0.5 & 0.7 & 1.2 \\
& 2.2 & 3.2 & 3.7 & 4.2 & \\
\hline
\end{tabular}

SWIR sensor is equipped with a thermoelectric cooler, the dark signal $S_{\mathrm{d}}$ in Fig. 6 is obviously still influenced by air temperature fluctuations.

Additionally, dark signal offsets $S_{\text {read }}$ exhibit a slight dependence on temperature in both spectrometers. The offsets $S_{\text {read }}$ between both sensors differ fundamentally with respect to their pixel-wise distribution and the fixed pattern noise they are creating. Figure 9 shows the noticeable uneven fixed pattern noise FPN ${ }^{\text {SWIR }}$ of the SWIR sensor. While FPN ${ }^{\text {VNIR }}$ appears smooth with $\sigma_{\mathrm{FPN}}^{\mathrm{VNIR}}=9.4 \mathrm{DN}$, the spatial distribution of FPN ${ }^{\text {SWIR }}$ is very uneven, with $\sigma_{\mathrm{FPN}}^{\mathrm{VNIR}}=173.8 \mathrm{DN}$. Bad pixels that were excluded in this analysis are also shown.

\subsubsection{Nonlinear radiometric response}

The photoelectric signal $\widetilde{S_{0}}$ from a perfectly linear sensor with response $R$ should scale linearly with the set integration time $t_{\text {set }}$ and radiance $L$ :

$\widetilde{S_{0}}=R L t_{\mathrm{set}}=s_{\mathrm{n}} t_{\mathrm{set}}$

Accordingly, there is an unambiguous normalized signal $s_{\mathrm{n}}=R L$ independent of camera settings for each radiance value $L$. However, measurements of constant radiance levels originating from a large integrating sphere (LIS) with various integration times (see Table 2) have shown deviations from this idealized linear model. This deviation between the idealized signal $\widetilde{S_{0}}$ and the actually observed signal $S_{0}$ is called photo response nonlinearity. Figures 7 and 8 show the deviations of the VNIR and SWIR found from the idealized linear model (Eq. 7). Here, the photoelectric signal $S_{0}$ of the same stabilized light source (LIS) should become invariant after normalization with the set integration time $t_{\mathrm{set}}$. The fit of the original VNIR signal $S_{0} / t_{\text {set }}$ (gray line, Fig. 7) shows a photo response nonlinearity. The nonlinearity at higher signal levels leads to a lower signal for $t_{\mathrm{set}}=12 \mathrm{~ms}$ compared with the signal for $t_{\mathrm{set}}=4 \mathrm{~ms}$. By contrast, the fit of the original SWIR signal $S_{0} / t_{\text {set }}$ (gray line, Fig. 8) is almost linear but is insufficiently normalized when using the set integration time $t_{\text {set }}$. To obtain absolute radiance values, the photoelectric signal $S_{0}$ has thus to be linearized first before the absolute radiometric response can be applied. In the following, the different deviations of both sensors from the linear model (Eq. 7) will be analyzed in detail.

We identified two effects which together explain the observed nonlinearities very well. According to Janesick (2007) 

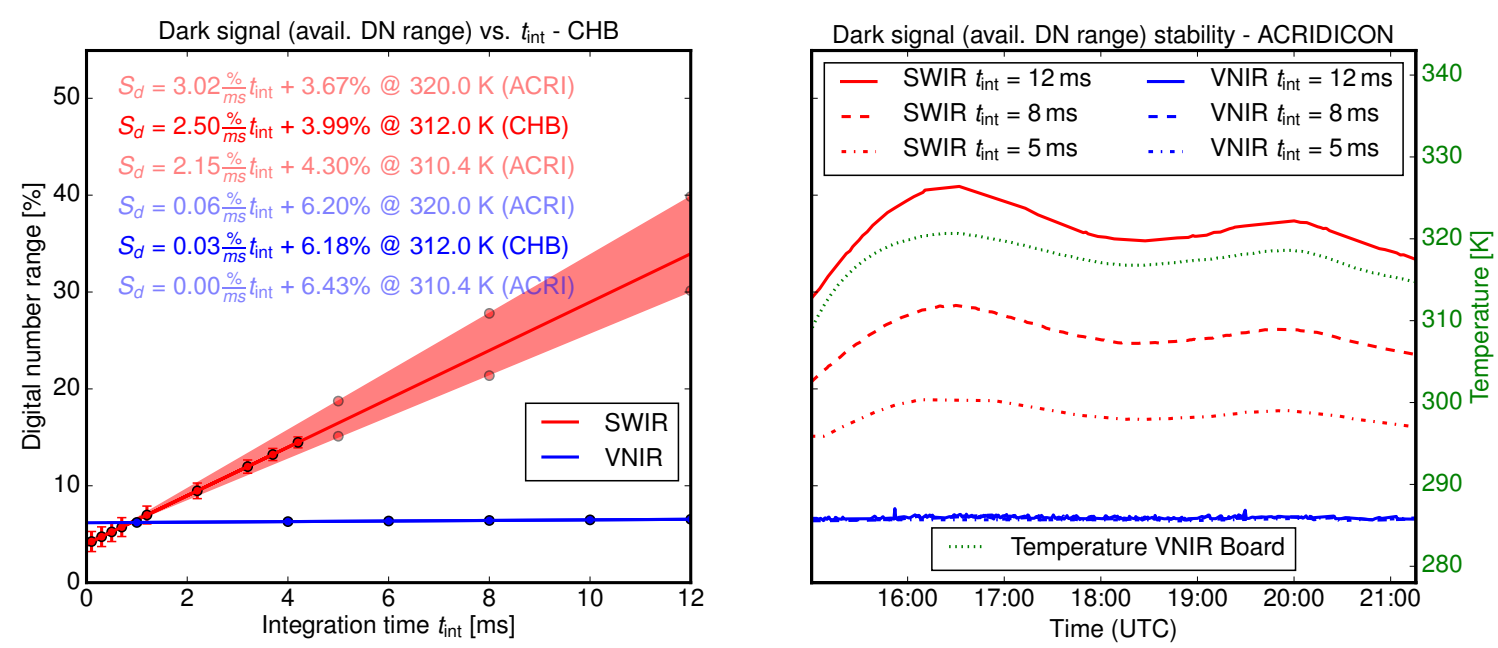

Figure 6. Left: mean dark signal levels $\overline{S_{\mathrm{d}}}$ in DN as a function of integration time $t_{\text {int }}$ when averaging over 500 dark frames as observed during the CHB calibration. Right: $\overline{S_{\mathrm{d}}}$ when averaging over 30 dark frames as observed on one flight AC14 (21 September 2014$)$ during the ACRIDICON-CHUVA campaign. The blue lines show $\overline{S_{\mathrm{d}}}$ for the VNIR, red lines for the SWIR spectrometer, while the different line styles denote different integration times. The green curve shows the temperature as measured within the VNIR casing. In both plots the dependence of $\overline{S_{\mathrm{d}}}$ from temperature and integration time becomes clearly visible for the SWIR, while $\overline{S_{\mathrm{d}}}$ remains constant for the VNIR.

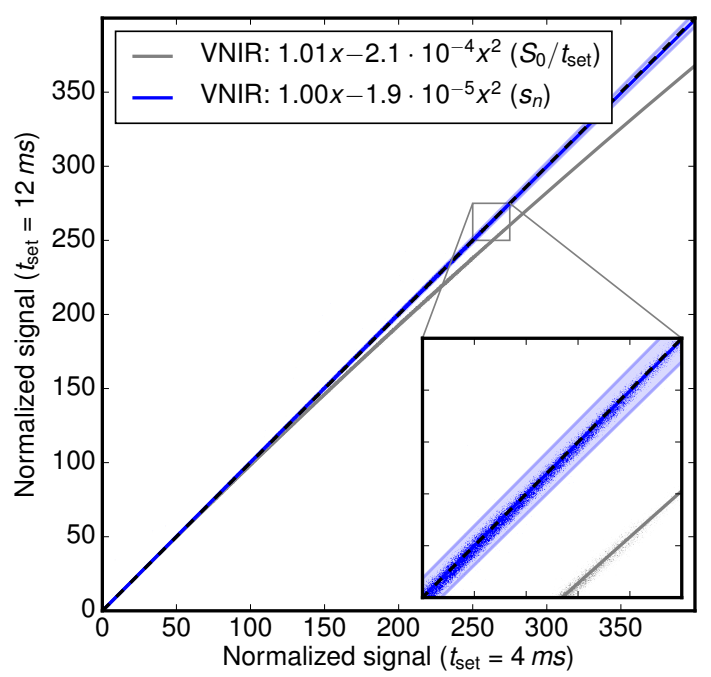

Figure 7. Integration time-normalized signal $S_{0} / t_{\text {set }}$ (gray line) of the stabilized light source (LIS), measured with the VNIR using two different integration times $t_{\mathrm{set}}=4 \mathrm{~ms}$ and $t_{\mathrm{set}}=12 \mathrm{~ms}$. The blue line shows the signal after nonlinearity correction $\left(s_{\mathrm{n}}\right)$ with the remaining nonlinearity uncertainty shown by the blue filled area. The dashed line represents the response of a perfectly linear sensor following Eq. (7).

the diode capacitance of CMOS detectors can increase significantly as charge collects. Thereby, the sensor-specific conversion gain $k[\mathrm{DN}]$ becomes a function of the number $N$ of received photoelectrons. For higher signal levels this causes a nonlinear relationship between incoming radiance $L$ and the photoelectric signal $S_{0}$. We considered this nonlinearity by adding a quadratic term to Eq. (7), which leads to the form

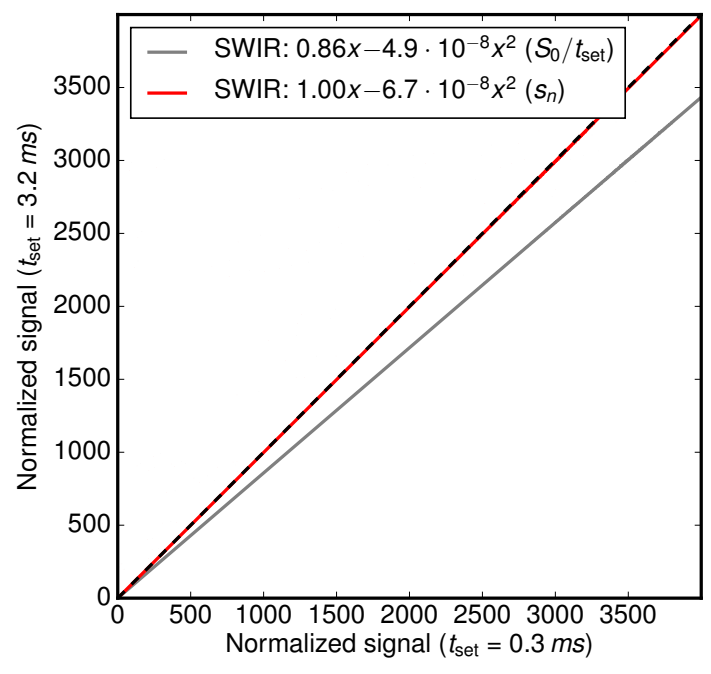

Figure 8. Integration time-normalized signal $S_{0} / t_{\mathrm{set}}$ (gray line) of the stabilized light source (LIS), measured with the SWIR using two different integration times $t_{\mathrm{set}}=0.3 \mathrm{~ms}$ and $t_{\mathrm{set}}=3.2 \mathrm{~ms}$. The red line shows the signal after normalization $\left(s_{\mathrm{n}}\right)$ using the corrected integration time $t_{\mathrm{set}}+0.055 \mathrm{~ms}$. The dashed line represents the response of a perfectly linear sensor following Eq. (7).

of Eq. (8). Furthermore, we found a small mismatch between the set integration time $t_{\text {set }}$ and the actual integration time $t_{\text {int }}$. For this reason, an offset term $t_{\mathrm{ofs}}$ was added to be fitted in the model in Eq. (8). The improved model which describes the observed photoelectric signal $S_{0}$ then reads

$S_{0}=s_{\mathrm{n}}\left(t_{\mathrm{set}}+t_{\mathrm{ofs}}\right)+\gamma\left(s_{\mathrm{n}}\left(t_{\mathrm{set}}+t_{\mathrm{ofs}}\right)\right)^{2}$. 


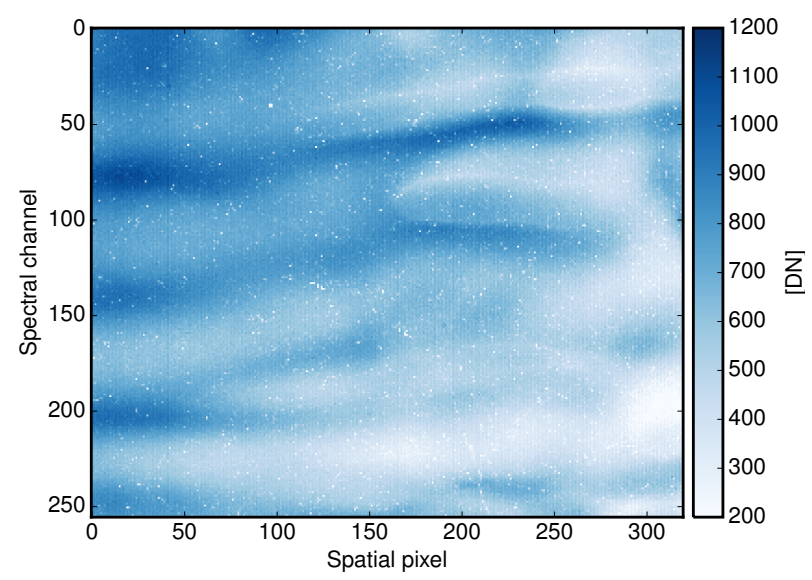

Figure 9. Extrapolated fixed pattern noise $\mathrm{FPN}_{\mathrm{SWIR}}$ in time at integration time $t_{\text {int }}=0 \mathrm{~s}$ for the SWIR spectrometer. The measurements were done with closed shutter at multiple integration times and reduced to $t_{\text {int }}=0 \mathrm{~s}$ by linear regression.

Table 3. Nonlinearity $\gamma$ and integration time offset $t_{\mathrm{ofs}}$ determined by fitting measurements to the model described in Eq. (8).

\begin{tabular}{lrl}
\hline Sensor & $\gamma\left[\mathrm{DN}^{-1}\right]$ & $t_{\mathrm{ofs}}(\mathrm{ms})$ \\
\hline VNIR & $(-2.3 \pm 0.3) 10^{-5}$ & $-0.001 \pm 0.01$ \\
SWIR & 0 & $+0.055 \pm 0.001$ \\
\hline
\end{tabular}

Here, $\gamma$ is the nonlinearity of $S_{0}$ in $\mathrm{DN}^{-1}$ and $t_{\mathrm{ofs}}$ is the offset between actual and reported integration time. The model can be inverted to yield the normalized signal $s_{\mathrm{n}}$ from measured signal $S$, dark signal $S_{\mathrm{d}}$ and $t_{\text {set }}$ when $\gamma$ and $t_{\text {ofs }}$ are known:

$s_{\mathrm{n}}=\frac{\sqrt{4 \gamma\left(S-S_{\mathrm{d}}\right)+1}-1}{2 \gamma\left(t_{\mathrm{set}}+t_{\mathrm{ofs}}\right)} \stackrel{\gamma \rightarrow 0}{\longrightarrow} \frac{S-S_{\mathrm{d}}}{t_{\mathrm{set}}+t_{\mathrm{ofs}}}$.

This nonlinear model converges to the linear model in Eq. (7) for $\gamma \rightarrow 0$ and $t_{\text {ofs }} \rightarrow 0$.

Using the integration times in Table 2 and the model described in Sect. 3.1.2, the parameters $\gamma$ and $t_{\text {ofs }}$ were determined for every pixel. This was done for measurements taken on the large integrating sphere at the CHB facility on 3 July 2014 by regression of measured $S_{0}$ on Eq. (8) using the least squares method. Since solar radiances are naturally very strong signals, the model in Eq. (8) was not designed to model effects at very low signal levels. For this reason, the fit was only done for pixels with signal levels higher than $2 \%$ of the maximum signal level. Mean and standard deviation of $\gamma$ and $t_{\text {ofs }}$ over the sensor are shown in Table 3. The fact that $\gamma$ and $t_{\mathrm{ofs}}$ do not vary much across pixels allows to use a single value for each of them on the whole sensor for simplicity. As the agreement between the presented model and all measurements was very good, a possible further dependence of the model parameters on other parameters has been discarded.

For the VNIR camera, the nonlinearity causes a deviation of $9 \%$ from the linear model at maximum signal level,

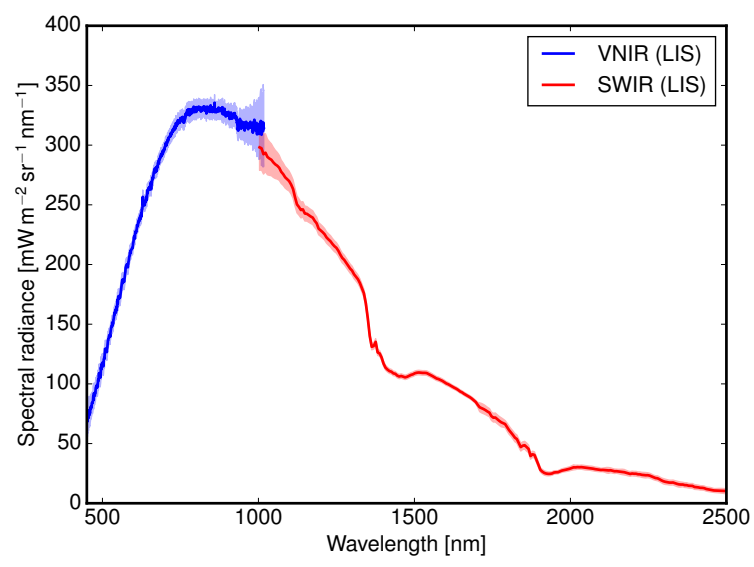

Figure 10. Reconstructed spectral radiance on top the large integrating sphere during the respective radiometric characterization. The absolute radiometric values were transferred from the RASTA base standard using the specMACS VNIR and SWIR sensors. The $2 \sigma$ uncertainty associated with the radiometric calibration is shown by the filled area.

while the SWIR camera does not exhibit a noticeable nonlinearity. In contrast, $t_{\mathrm{ofs}}$ of the VNIR camera is negligible with $0.001 \mathrm{~ms}$ while the SWIR offset $0.055 \mathrm{~ms}$ lies within the same order of magnitude as the shortest possible integration time of $0.1 \mathrm{~ms}$.

By using the found parameters $\gamma$ and $t_{\mathrm{ofs}}$ in the nonlinear model (Eq. 9), the linearized signal $s_{\mathrm{n}}$ of the VNIR is shown by the blue line in Fig. 7, while $s_{\mathrm{n}}$ of the SWIR is shown by the red line in Fig. 8. After the nonlinearity correction, the VNIR signal $s_{\mathrm{n}}$ better follows the linear model. Likewise, the corrected SWIR signal $s_{\mathrm{n}}$ now seems sufficiently normalized by using the additional integration time offset $t_{\text {ofs }}$.

The uncertainty in $\gamma$ and $t_{\text {ofs }}$ leads to a remaining nonlinearity uncertainty $\sigma_{\text {nonlin }}$. The maximum error due to this uncertainty was estimated by using the error boundaries of both parameters in Eq. (9). Only the uncertainty in $\gamma$ for the VNIR is of significance which is shown by the blue filled area in Fig. 7.

During this analysis, some alternative nonlinearity models have been tested in place of the existing nonlinearity parameterization, which is assumed to be a function of total collected radiative energy $\left(\propto L \cdot t_{\text {int }} \propto s_{\mathrm{n}} \cdot t_{\mathrm{int}}\right)$. A simpler model, considering only a quadratic term in $t_{\text {int }}$, was not able to provide similarly good results as the model presented above. Some combinations of quadratic or higher order terms in the form of $s_{\mathrm{n}}^{a} \cdot t_{\text {int }}^{b}$ have also been tried, assuming equal nonlinear response of all pixels of one sensor and exploiting the intensity variations between pixels as introduced by the spectrograph. As the assumption of equal nonlinear response for all pixels has been found to hold true for the model that was finally chosen and neither of the alternate models showed better results, they have also been discarded. This behavior suggests, but is no evidence, that the signal is actually a non- 
linear function of the total collected radiative energy and neither in $t_{\text {int }}$ nor $L$ alone.

\subsubsection{Absolute radiometric response}

After nonlinearity correction, the normalized signal $s_{\mathrm{n}}$ in $\left[\mathrm{DN} \mathrm{ms}{ }^{-1}\right]$ can be converted into absolute radiance values $L$ in $\left[\mathrm{mWm}^{-2} \mathrm{~nm}^{-1} \mathrm{sr}^{-1}\right]$. Using the absolute radiometric response $R$ in $\left[\mathrm{DN} \mathrm{ms}^{-1} \mathrm{~mW}^{-1} \mathrm{~m}^{2} \mathrm{~nm} \mathrm{sr}\right.$ ], this can be described by

$L=R^{-1} \cdot s_{\mathrm{n}}$.

$R$ is different for each pixel and thereby also covers the correction of inter-pixel variations of the sensor response, also called photo response nonuniformity (PRNU). The absolute radiometric response $R$ is determined once during a radiometric calibration with a known radiance standard.

In this work, the absolute radiometric response $R$ was characterized using the absolute RAdiance STAndard (RASTA; Schwarzmaier et al., 2012) of the IMF at DLREOC. In turn, the RASTA was characterized with absolute radiance standards operated by the PTB (PhysikalischTechnische Bundesanstalt), the German National Metrology Institute. As the RASTA does not cover the full field of view of the sensors, a large integrating sphere (LIS) was additionally used as an isotropic light source. As determined by Baumgartner (2013), the output stability of the LIS is better than $\sigma=0.02 \%$ for a duration of $330 \mathrm{~s}$.

To transfer the absolute radiance standard from the RASTA to the LIS, measurements of both light sources were performed in fast succession with pixels in the geometric center of the specMACS FOV. The absolute calibration of the RASTA can then be transferred to the LIS by using the ratio between the normalized signals $s_{\mathrm{n} \text {;LIS }}$ and $s_{\mathrm{n} \text {;RASTA }}$ measured at the integrating sphere and the absolute standard:

$L_{\mathrm{LIS}}=L_{\mathrm{RASTA}} \cdot \frac{L_{\mathrm{LIS}}}{L_{\mathrm{RASTA}}}=L_{\mathrm{RASTA}} \cdot \frac{s_{\mathrm{n} ; \mathrm{LIS}}}{s_{\mathrm{n} ; \mathrm{RASTA}}}$.

Simultaneously the calibration transfer was done with a second, independent spectrometer (SVC HR-1024i) to validate the transfer with the specMACS instrument. In Fig. 10 the spectral radiance of the large integrating sphere is shown as it was transferred from the RASTA standard using Eq. (11).

With the LIS illuminating the complete FOV of the instrument the conversion from normalized signals to absolute radiances ( $R$ in Eq. 10) is calculated for each pixel.

The absolute radiometric response $R$ of the VNIR and the SWIR sensors are presented in Fig. 11. Both sensors show strongly reduced sensitivity at the upper and lower boundaries of the spectrum. This is expected due to the nature of the material-dependent band gap and the transmissivity of the optical system. The VNIR sensor shows an etalon fringe pattern (seen in Fig. 11 (left) along the spectral dimension) typical for backside-illuminated sen- sors (Marques Vatus and Magnan, 2004), whereas the frontilluminated SWIR sensor does not exhibit significant patterns. The discrepancy between the absolute radiometric responses, $R$, that were found and the ones given by the manufacturer does not exceed more than $10 \%$.

Using the nominal accuracies of the reference light sources and signal statistics derived from the sensors during characterization measurements, an error budget for the absolute radiometric response $R$ was calculated and is shown as $2 \sigma$ uncertainty in Fig. 12. The uncertainty in the absolute radiometric calibration of the RASTA was given by the PTB and is indicated by the green line in Fig. 12. At longer wavelengths, the nominal uncertainty of the RASTA increases. This can be traced back to the accuracy of the reference radiometers used during the RASTA characterization at the PTB. The uncertainty due to inhomogeneities of the LIS is given to be $\pm 1.6 \%$ (Baumgartner, 2013). Another source of uncertainty arises from the drift of the dark signal $S_{\mathrm{d}}$ over time and from the noise $\mathcal{N}$ of the signal $S$, which is shown as blue (VNIR) and red (SWIR) dashed lines. The drift per minute was assumed to be $10 \mathrm{DN}$ for the SWIR and $3 \mathrm{DN}$ for the VNIR as found in the dark signal analysis (Sect. 3.1.1). The noise was calculated for 100 averaged dark frames and 500 averaged illuminated frames resulting in $\sigma_{\langle S\rangle}=0.5 \mathrm{DN}$. At the RASTA and the LIS the $2 \sigma$ uncertainty due to the dark signal drift and noise accounts to around $1 \%$ for the VNIR and 3\% for the SWIR for wavelengths in the center of both spectra. While dark signal drift and noise level stay constant with wavelength, radiometric sensitivity and signal $S$ decrease towards the edges of the spectra. This results in a sharp increase of the relative uncertainty towards spectral regions with low radiometric sensitivity. Especially towards the edges of the spectra, the drift of the dark signal $S_{\mathrm{d}}$ contributes the most to the overall radiometric uncertainty. Altogether this error budget is a very conservative estimate for an individual measurement with a single pixel without any averaging. For relative measurements, some of these uncertainties cancel out e.g., the LIS inhomogeneity and the RASTA uncertainty during a window transmissivity characterization presented in Sect. 4.2.1.

\subsubsection{Noise}

The noise $\mathcal{N}$ is composed of dark current noise $\mathcal{N}_{\mathrm{dc}}$, read noise $\mathcal{N}_{\text {read }}$, and photon shot noise $\mathcal{N}_{\text {shot }}$. Their joint standard deviation $\sigma_{\mathcal{N}}$ is calculated using the individual standard deviations $\sigma_{\text {shot }}, \sigma_{\mathrm{dc}}$ and $\sigma_{\text {read }}$ :

$\sigma_{\mathcal{N}}=\sqrt{\sigma_{\text {shot }}^{2}+\sigma_{\mathrm{dc}}^{2}+\sigma_{\text {read }}^{2}}=\sqrt{k^{2} N+\sigma_{\mathrm{d}}^{2}}$.

Since photons arrive randomly in time, the number $N$ of photoelectrons measured during a fixed time interval is distributed according to the Poisson distribution. Following the Poisson statistics the standard deviation $\sigma_{N}$ of a distribution with the expectation value $N$ is proportional to $\sqrt{N}$. For this 

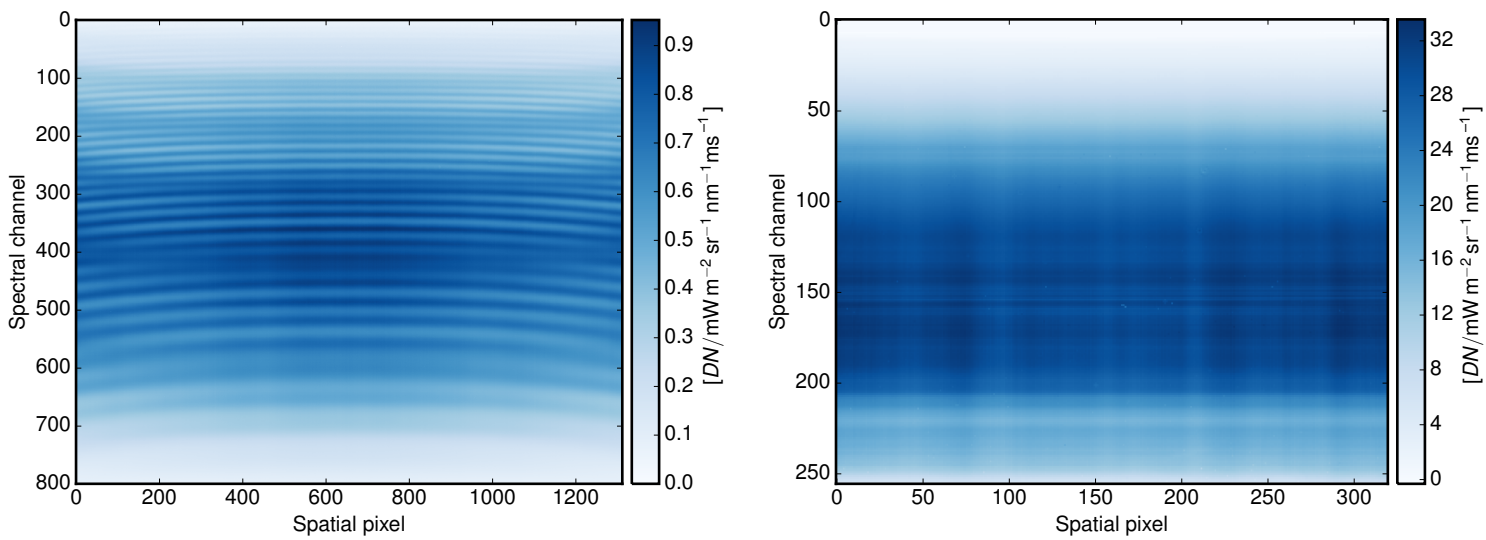

Figure 11. Absolute radiometric response $R$ in $\left[\mathrm{DN} \mathrm{ms} \mathrm{mW}^{-1} \mathrm{~mW}^{-1} \mathrm{~nm} \mathrm{sr}\right]$ for the VNIR (left) and SWIR (right) spectrometer. The radiometric response shows a strong dependence with wavelength for both sensors, which is expected due to a material-dependent band gap and the specific transmissivity of the optical system. The VNIR sensor shows an etalon fringe pattern typical of backside-illuminated sensors.

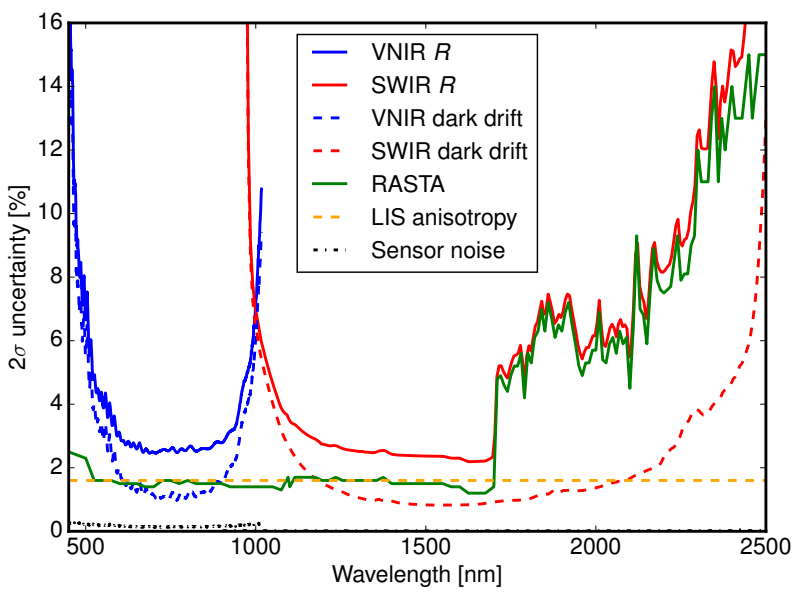

Figure 12. Main contributions to the $2 \sigma$ uncertainty of the absolute radiometric response $R$. The uncertainties resulting from sensor noise and dark current drift are shown for the RASTA measurements. Due to the lower radiance of the RASTA, other noise and drift components contribute less to the total error.

reason, the photon shot variance $\sigma_{\text {shot }}^{2}$ scales linearly with the number of incoming photoelectrons $N$ and the squared conversion gain $k^{2}\left[\mathrm{DN}^{2}\right]$ (Eq. 12). For a sensor with linear response, the relation $S_{0} \propto N$ holds. A deviation from this relationship can be an indication of a nonlinear relationship between the number $N$ of photoelectrons and the photoelectric signal $S_{0}$ or is caused by a non-Poisson noise source.

Similar to the dark signal $S_{\mathrm{d}}$ in $S$, a dark noise $\mathcal{N}_{\mathrm{d}}$ remains in $\mathcal{N}$ without illumination. It comprises dark current noise $\mathcal{N}_{\text {dc }}$ and read-out noise $\mathcal{N}_{\text {read }}$. The dark current noise is caused by statistical variation of thermally generated electrons. Pixel readout and analog to digital conversion are further subject to electronic read-out noise, which is independent of integration time.
For this analysis, $\sigma_{\mathcal{N}}$ is calculated as the pixel-wise standard deviation of 500 consecutive frames which were obtained during the nonlinearity measurements with varying integration times as listed in Table 2. Here, the noise standard deviation $\sigma_{\mathcal{N}}$ and mean, dark-current-corrected signal level $\left\langle S_{0}\right\rangle$ are calculated individually for each pixel, since both sensors cannot be homogeneously illuminated due to the spectrographic diffraction grating. As noise $\mathcal{N}$ describes unbiased temporal variations of the signal around its expectation value, its temporal mean vanishes: $\langle\mathcal{N}\rangle \approx 0$.

Figure 13 shows the results of the noise analysis. For each pixel and each integration time, the mean and standard deviation were calculated and accumulated in the shown 2-D histograms. The noise characteristics of the VNIR are shown on the left while results for the SWIR are shown on the right. On the top in Fig. 13 the pixel-wise standard deviation $\sigma_{\mathcal{N}}$ is plotted against the mean, dark-current-corrected signal level $\left\langle S_{0}\right\rangle$ on a log-log scale. On the bottom, the same is done with the pixel-wise variance $\sigma_{\mathcal{N}}^{2}$ on a linear scale.

With the classic photon transfer curve (Janesick, 2007) the noise characteristics can be used to determine many important camera parameters. When the signal noise standard deviation $\sigma_{\mathcal{N}}$ is plotted against the mean, dark-current-corrected signal level $\left\langle S_{0}\right\rangle$ on a log-log scale, like it is done in Fig. 13, different noise regimes become apparent. The dark noise at integration time $t_{\text {int }}=0 \mathrm{~s}$ at the lower end of $\left\langle S_{0}\right\rangle$ is dominated by read-out noise with standard deviation $\sigma_{\text {read }}$. With increasing mean signal level $\left\langle S_{0}\right\rangle$ photon shot noise becomes dominant with $\sigma_{\text {shot }}$. Due to the Poisson-like distribution the photon shot noise variance $\sigma_{\text {shot }}^{2}$ should scale linearly with mean signal level. Deviations from this linear relationship can provide an indication of a nonlinear radiometric response (Bohndiek et al., 2008) or a charge-sharing mechanism between pixels (Downing et al., 2006; Stefanov, 2014).

At low values of $\left\langle S_{0}\right\rangle$ the signal-independent read-out noise becomes apparent. The read-out noise for $t_{\mathrm{int}}=0 \mathrm{~s}$ is 

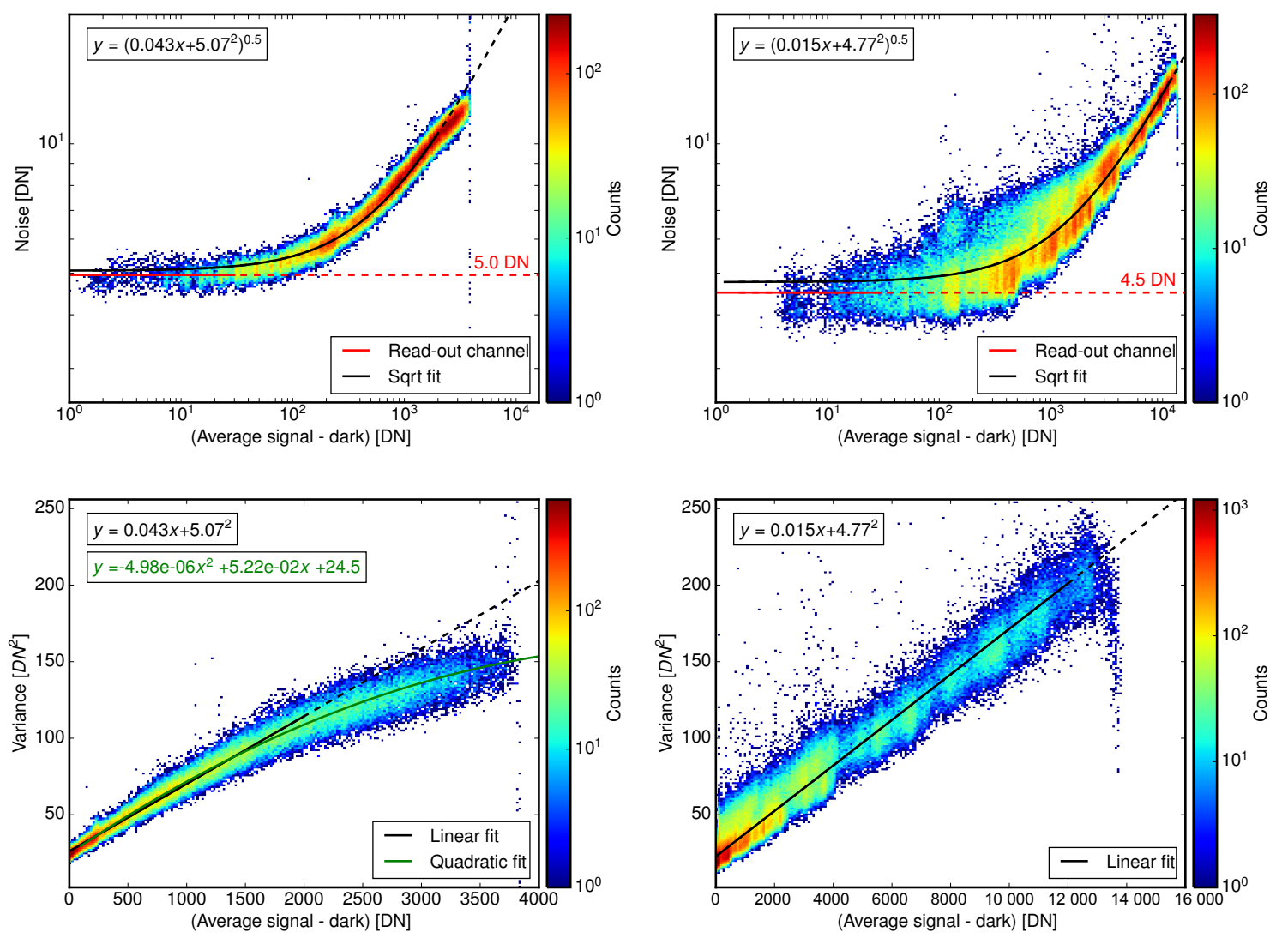

Figure 13. Noise characteristics for the (left) VNIR and (right) SWIR spectrometer. Top: the noise $\sigma_{\mathcal{N}}$ (standard deviation of $S$ ) is shown against the mean, dark-current-corrected signal level $\left\langle S_{0}\right\rangle$. Bottom: noise variance $\sigma_{\mathcal{N}}^{2}$ against $\left\langle S_{0}\right\rangle$ for both spectrometers.

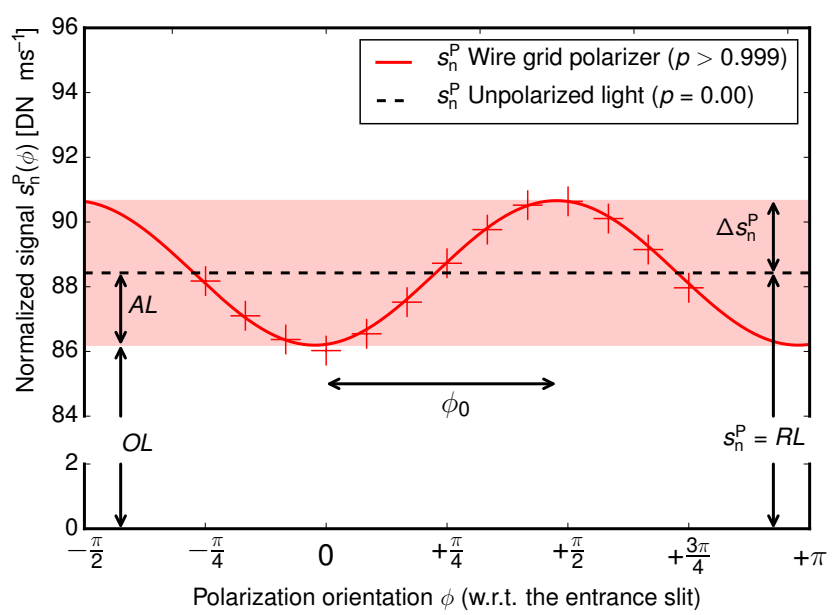

Figure 14. Polarization-dependent normalized photoelectric signal $s_{\mathrm{n}}^{\mathrm{P}}(\phi)$ for one VNIR pixel (spatial: 400 , spectral: 600 ) while rotating the polarizer between -45 and $135^{\circ}$ with respect to the entrance slit in steps of $15^{\circ}$. The polarization sensitivity $P$ and its orientation $\phi_{0}$ is found by fitting the measurements with the model in Eq. (16).

derived from the $y$-intercept of a constant fit on $\sigma_{\mathcal{N}}$ for $\left\langle S_{0}\right\rangle<30$ DN. By doing this, the noise associated with the read-out channel was found to be 5.0DN for the VNIR and 4.5 DN for the SWIR spectrometer. For larger values of $\left\langle S_{0}\right\rangle$ the noise begins to increase.

When the standard deviation $\sigma_{\mathcal{N}}$ is fitted with the square root model following Eq. (12), the noise characteristics can be further investigated. At first glance, the noise standard deviation of the VNIR sensor is in accordance with the noise model described by Eq. (12) with a constant conversion gain $k=0.043[\mathrm{DN}]$. For values of $S_{0}$ between 0 and $2000 \mathrm{DN}$ it follows the function

$\sigma_{\mathcal{N}}=\sqrt{0.043 S_{0}+5.07^{2}}[\mathrm{DN}]$

For larger values the noise variance $\sigma_{\mathcal{N}}^{2}$ no longer scales linearly with $\left\langle S_{0}\right\rangle$ but remains below the fit in Eq. (13). As seen in Fig. (13) bottom left, the VNIR noise can no longer be explained by a Poisson noise model (Eq. 12) with a constant conversion gain $k$ [DN]. This noise characteristic can be an indication of two different mechanisms at work. Either $k$ [DN] varies with signal level $S_{0}$, which would cause a photon response nonlinearity; or a charge sharing is occurring between pixels, which would violate the Poisson assumption. A more in-depth analysis of the noise showed a small autocorrelation between pixels of the same spatial sensor row, 

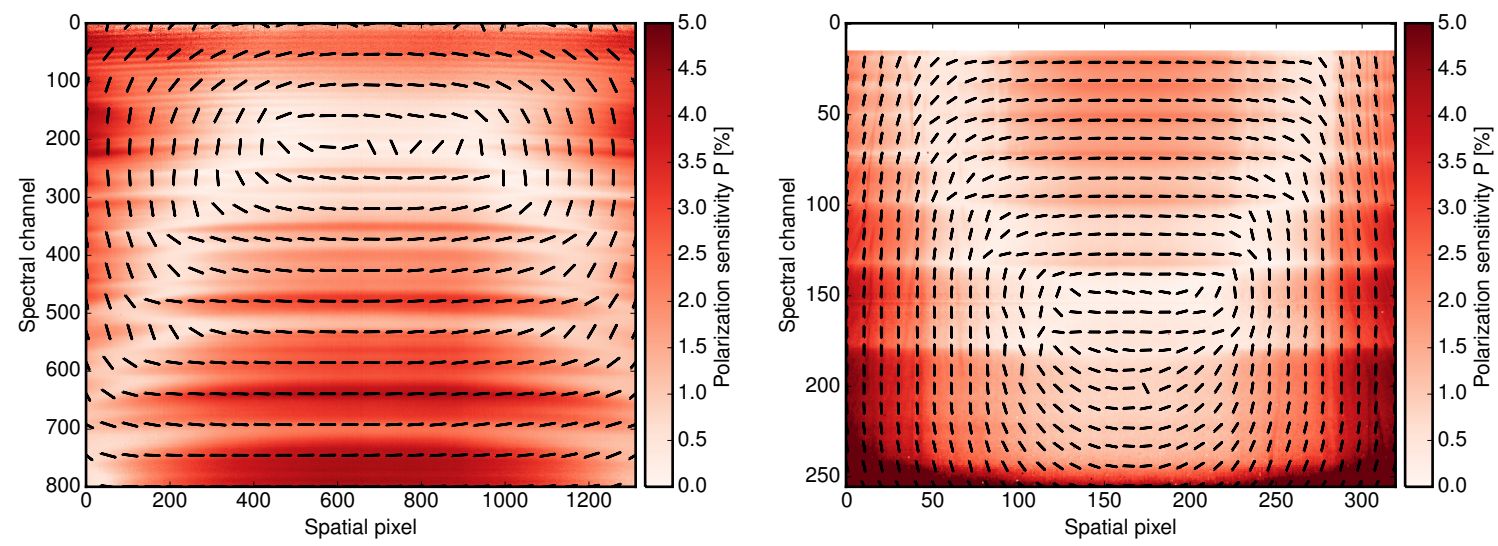

Figure 15. Results of the polarization sensitivity characterization for the VNIR (left) and the SWIR (right). The color map shows the polarization sensitivity $P$ for all pixels as determined with Eq. (17). With the entrance slit oriented horizontally to this figure, the black solid lines indicate the polarization orientation for which the signal becomes minimal.

which would suggest the latter; but as the photon response nonlinearity analysis in Sect. 3.1.2 has shown, radiometric nonlinearity has to be considered as a possible explanation, too.

In contrast, the SWIR noise standard deviation $\sigma_{\mathcal{N}}$ shown in the top right of Fig. 13 compares much better to the Poisson model. Between 0 and $12000 \mathrm{DN}$, which is only limited by the subtracted dark signal, it fits the following form:

$\sigma_{\mathcal{N}}=\sqrt{0.015 S_{0}+4.77^{2}}[\mathrm{DN}]$.

At larger values of $\left\langle S_{0}\right\rangle$, the noise variance $\sigma_{\mathcal{N}}^{2}$ remains linear with $k=0.015$ [DN] until saturation is reached. For both sensors, no wavelength dependence in noise was found.

\subsubsection{Polarization sensitivity}

All optical components can exhibit polarization-dependent loss, which in effect makes the signal sensitive to polarization. This polarization sensitivity has an influence on the absolute radiometric response $R$ when parts of the measured light are linearly polarized. The polarization sensitivity can be examined by splitting the instrument response virtually into a polarization insensitive part with partial response $O$ and a polarization sensitive part with partial response $2 A$, such that $R=A+O$ for unpolarized light. In line with Malus' Law the polarization-dependent normalized photoelectric signal $s_{\mathrm{n}}^{\mathrm{P}}(\phi)$ of incoming radiance $L$, with a degree of polarization $p$, measured with such an instrument is given by

$$
\begin{aligned}
s_{\mathrm{n}}^{\mathrm{P}}(\phi) & =2 A \cdot L_{\|}+O \cdot L \\
& =2 A\left(p \cos ^{2}\left(\phi-\phi_{0}\right)+\frac{1-p}{2}\right) \cdot L+O \cdot L .
\end{aligned}
$$

Here, $L_{\|}$denotes the incoming radiation parallel to the sensor's polarization direction, $\phi$ the polarization orientation with respect to the entrance slit, and $\phi_{0}$ the polarization orientation for which $s_{\mathrm{n}}^{\mathrm{P}}(\phi)$ is maximal.

To investigate the polarization influence, a wide-band wire grid polarizer $(99.9 \%$ degree of polarization between 400 and $2500 \mathrm{~nm}$ ) mounted on a rotation stage was placed between the large integrating sphere and the specMACS instrument. Following Lenhard et al. (2015), measurements of the photoelectric signal $s_{\mathrm{n}}^{\mathrm{P}}(\phi)$ were done while rotating the polarizer between 0 and $180^{\circ}$ with respect to the entrance slit in steps of $15^{\circ}$. For fully polarized light $(p=1)$ of intensity $L$, Fig. 14 shows the polarization sensitive behavior of $s_{\mathrm{n}}^{\mathrm{P}}(\phi)$ for one VNIR pixel (spatial: 400, spectral: 600) while rotating the wire grid polarizer (red crosses). While the maximum of $s_{\mathrm{n}}^{\mathrm{P}}(\phi)$ can be found for polarization orientations parallel to $\phi_{0}$, the maximum signal loss due to the polarization sensitivity occurs orthogonal to $\phi_{0}$

In the following, the polarization sensitivity $P$ is defined as the increase of the signal between unpolarized light ( $p=$ 0 ) and light fully polarized in the most sensitive direction of the sensor $\left(p=1, \phi=\phi_{0}\right)$, while the total radiance of the light source remains unchanged. The polarization sensitivity $P$ reads as follows:

$P=\frac{A}{A+O} \cdot 100 \%$.

A natural light source has an unknown degree $p$ and orientation $\phi$ of polarization. Nonetheless, the maximum error in the normalized signal $s_{\mathrm{n}}$ due to polarization can be given for an estimated maximum degree of polarization $p_{\max } \leq 1$. Note that this estimate is always possible in the form of $p_{\max }=1$ for a completely unknown light source. Following Eq. (16), any signal $s_{\mathrm{n}}^{\mathrm{P}}$ measured from an incoming radiance $L$ with maximum degree of polarization $p_{\max }$ can be constrained for the following bounds (which are illustrated by the red shaded region in Fig. 14):

$\left(\left(1-p_{\max }\right) A+O\right) L \leq s_{\mathrm{n}}^{\mathrm{P}} \leq\left(\left(1+p_{\max }\right) A+O\right) L$. 
Ideally, the signal would be independent of $\phi$, following the linear model $s_{\mathrm{n}}=R L$. In particular, this holds true for $s_{\mathrm{n}}^{\mathrm{P}}$ for unpolarized light $\left(p_{\max }=0\right)$, as it was the case during the radiometric characterization. It follows that the error $\Delta s_{\mathrm{n}}$ for an unknown degree $p>0$ and orientation $\phi$ of polarization is given by $\Delta s_{\mathrm{n}}=\left|s_{\mathrm{n}}-s_{\mathrm{n}}^{\mathrm{P}}\right|$. An upper bound of the error $\Delta s_{\mathrm{n}}$ due to polarization can then be estimated by using $s_{\mathrm{n}}=R L$, $R=A+O$, and Eq. (18):

$\Delta s_{\mathrm{n}} \leq \max \left(\left|R L-\left(\left(1 \pm p_{\max }\right) A+O\right) L\right|\right)=p_{\max } A L$.

Furthermore, an upper bound for the relative uncertainty due to polarization can be estimated using the polarization sensitivity $P$ by estimating $L$ through $s_{\mathrm{n}}^{\mathrm{P}}$ using Eq. (18) again and inserting Eq. (17) after solving for $A$ :

$$
\frac{\Delta s_{\mathrm{n}}}{s_{\mathrm{n}}^{\mathrm{P}}} \leq \frac{p_{\max } A}{\left(1-p_{\max }\right) A+O}=\frac{p_{\max } P}{1-p_{\max } P} .
$$

In the field, radiation is never fully polarized. The polarization of sunlight reflected by water clouds is well below $5 \%$ for most viewing geometries. It only reaches values of up to $15 \%$ in the rainbow region of optically very thin clouds (Hansen, 1971). In contrast, Rayleigh scattering can be strongly polarized, depending on the scattering angle. If strongly polarized light must be assumed, the calibrated radiance has to be handled with care and provided with corresponding uncertainty estimates following Eq. (20). For sensor regions with a small polarization sensitivity $P$, the relative radiometric error due to polarization scales linearly with the light polarization $p$.

The polarization sensitivity $P$ and the angular offset $\phi_{0}$ were found by fitting the measurements to Eq. (16). In Fig. 15, the characterization results for $P$ and $\phi_{0}$ are shown as color and black lines respectively. Here, the black solid lines indicate the polarization orientation for which the signal becomes minimal. The polarization sensitivity $P$ can be observed to increase from 1 to $5 \%$ towards larger wavelengths for both cameras, resulting in a maximum error of $5.3 \%$ for fully polarized light. While $P$ is higher in the center of the VNIR FOV, it increases towards the edges for the SWIR. Furthermore, very high values for $P(>5 \%)$ can be observed at both wavelength cutoffs of the SWIR, where the radiometric sensitivity becomes very small. Due to the very low radiometric sensitivity of the SWIR, the region of the shortest wavelengths was excluded in this analysis. Despite the slightly different definition, the values of $P$ agree well with Lenhard et al. (2015) and Hyvarinen et al. (1998) and can be explained by the polarization caused at the entrance slit and the holographic transmission grating.

\subsubsection{Overall radiometric uncertainty budget}

To specify the total radiometric uncertainty for every measurement, the following section will give a bottom-up cal- culation of the propagation of radiometric errors. As it has already been done during the estimation of the total dark signal uncertainty, maximum errors $(\Delta)$ are being used as an approximation of $2 \sigma$ errors, when no standard deviation is available.

First, the absolute error contributions to the photoelectric signal $S_{0}$ are combined:

$2 \sigma_{S_{0}}=\sqrt{\left(2 \sigma_{\mathrm{d}}\left(t_{0}\right)\right)^{2}+\left(2 \sigma_{\mathcal{N}}\left(S_{0}\right)\right)^{2}}$.

Here, $\sigma_{\mathrm{d}}\left(t_{0}\right)$ denotes the estimated standard deviation of the dark signal (following Eq. 6) and $\sigma_{\mathcal{N}}\left(S_{0}\right)$ is the estimate of the instantaneous noise of the signal (derived from the photon transfer curve). Subsequently, the relative error of the normalized signal is obtained by combining the relative errors of the photoelectric signal $\sigma_{S_{0}}$ with the estimated remaining nonlinearity uncertainty $\sigma_{\text {nonlin }}$ and the polarization uncertainty $\Delta s_{\mathrm{n}}$ :

$\frac{2 \sigma_{S_{\mathrm{n}}}}{s_{\mathrm{n}}}=\sqrt{\left(\frac{2 \sigma_{S_{0}}}{S_{0}}\right)^{2}+\left(\frac{2 \sigma_{\text {nonlin }}}{s_{\mathrm{n}}}\right)^{2}+\left(\frac{\Delta s_{\mathrm{n}}}{s_{\mathrm{n}}^{\mathrm{P}}}\right)^{2}}$.

Lastly, radiometric calibration additionally adds the uncertainty $\frac{\sigma_{R}}{R}$ of the sensor response:

$$
\frac{2 \sigma_{L}}{L}=\sqrt{\left(\frac{2 \sigma_{s_{\mathrm{n}}}}{s_{\mathrm{n}}}\right)^{2}+\left(\frac{2 \sigma_{R}}{R}\right)^{2}} .
$$

An example of typical total uncertainty values for real measurements is given later in the application section (Sect. 4.2.2).

\subsection{Spatial and spectral characterization}

Besides the radiometric characterization of the spectrometer, its spatial and spectral projection onto the detector are of great importance for the scientific application. The radiance contribution for a single pixel from different solid angles is described by two line spread functions (LSFs), the acrossand along-track LSFs. The spectral responsivity for every pixel is described by a spectral response function (SRF). Moreover, some pixels of the detector yield unreliable (dead pixel) or biased (hot pixel) measurements. These should be marked and classified as "bad".

\subsubsection{Bad pixel correction}

Bad pixels do not behave according to the instrument model assumed by the calibration procedure. As argued by Lenhard et al. (2015), bad pixel characterization of an assembled hyperspectral sensor is not straight forward as a uniform illumination of the sensor chip is not achievable due to the dispersing element. It was decided to manually observe measured data over time. Pixels behaving very differently from surrounding pixels are collected in a list associated with the 

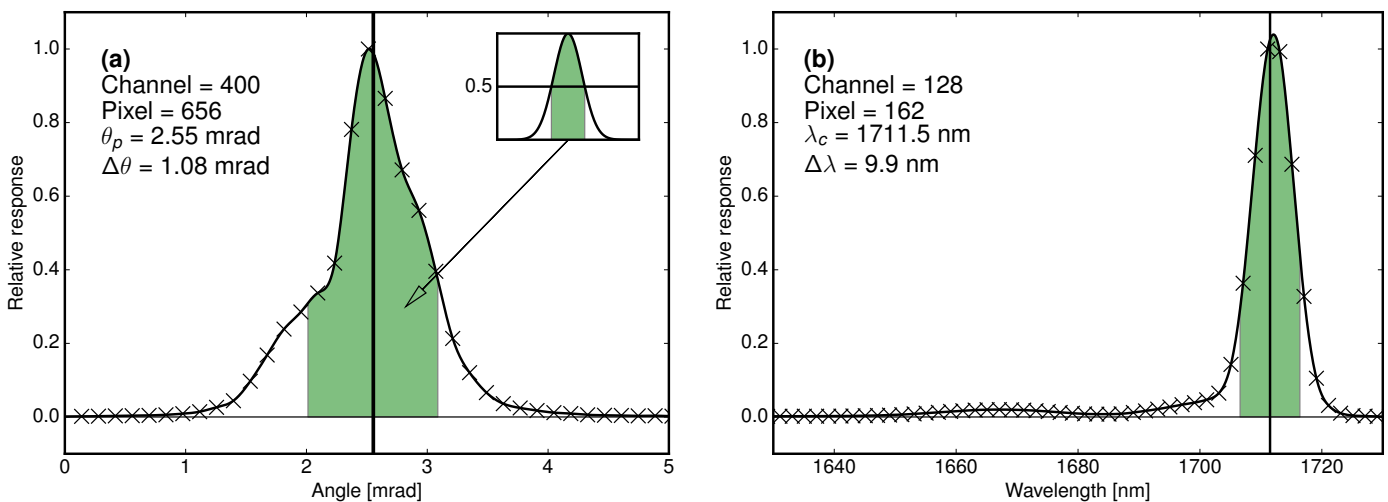

Figure 16. (a) LSF of spectral channel 400 for spatial pixel 656 of the VNIR sensor. The vertical line denotes the median viewing angle $\theta_{c}$, the width of the filled area the angular resolution $\Delta \theta$. (b) SRF of spectral channel 128 for spatial pixel 162 of the SWIR sensor. The vertical line denotes the median wavelength $\lambda_{c}$, the width of the filled area the wavelength bandwidth $\Delta \lambda$. For both plots the crosses are the measurements, and the curve is a spline fitted to these data points.
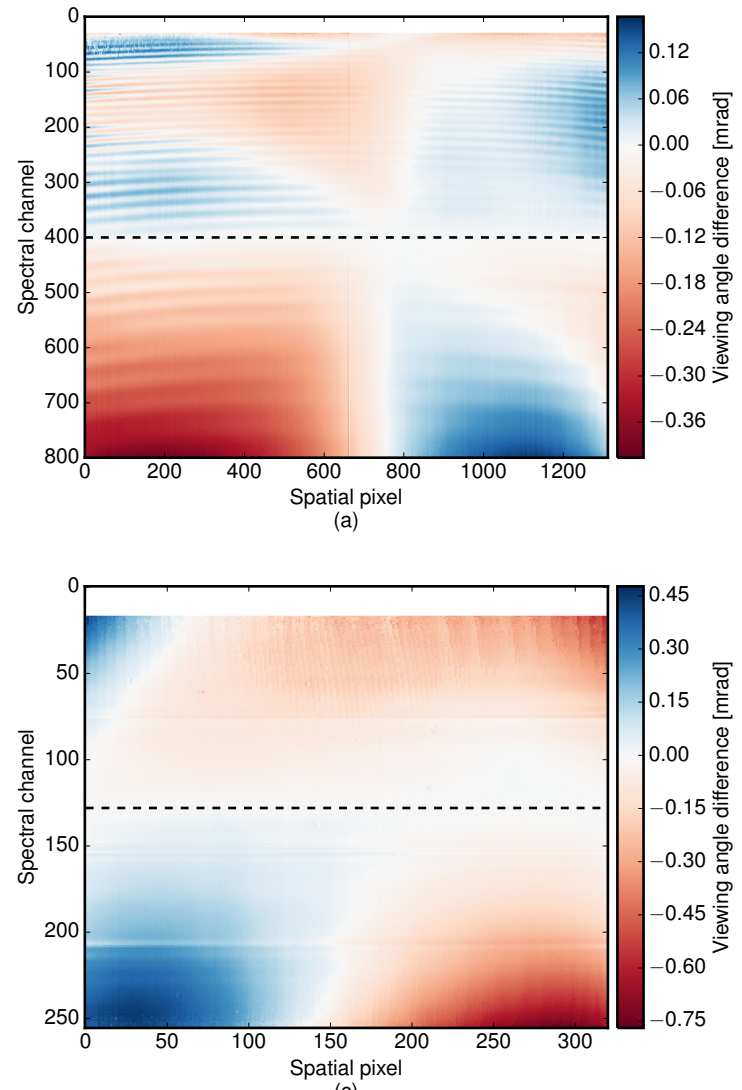

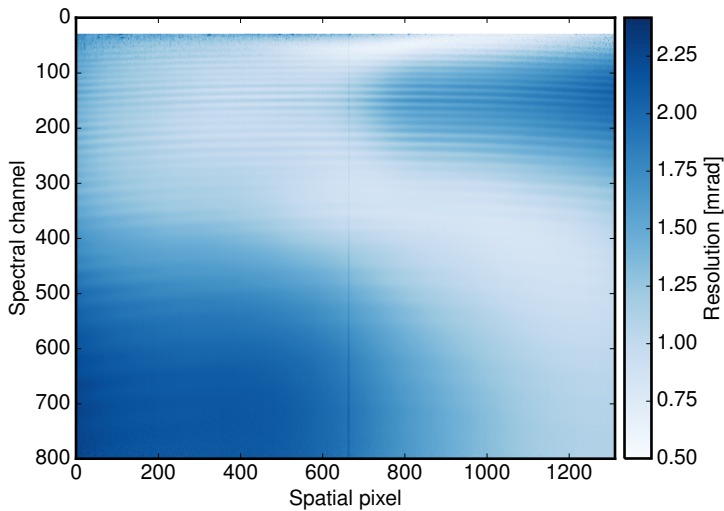

(b)

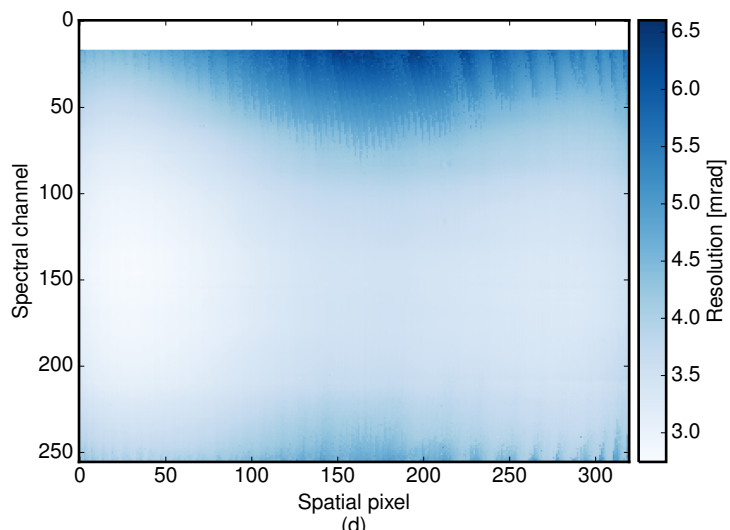

(d)

Figure 17. Spatial across-track characterization results of the VNIR (top) and SWIR (bottom) sensor. Panels (a) and (c) show the difference of the viewing angle of each pixel to those of channel 400 (VNIR) and channel 128 (SWIR) respectively. Panels (b) and (d) show the angular resolution. White areas indicate channels which are excluded from evaluation due to their low sensitivity.

calibration files. For the VNIR sensor, there was no previous knowledge about bad pixels. For the SWIR sensor, the list of bad pixels provided by the manufacturer was included. Currently, one bad pixel is known for the VNIR sensor and
264 randomly distributed bad pixels are known for the SWIR sensor.

Bad pixel correction or the replacement of invalid pixel values by interpolated values is needed if further processing algorithms cannot handle invalid pixels in the resulting data 
set. Depending on the goal of the proceeding analysis, different interpolation schemes may be appropriate. Currently, bad pixel correction is implemented based on the list of bad pixels provided by the calibration file and a user-defined strategy how interpolation rules should be derived from the bad pixel list. The primarily used strategy is to perform a linear interpolation from spatially adjacent good pixels over a single bad pixel or a group of bad pixels in order to keep spectral features intact.

\subsubsection{Response function}

Figure 16 shows a measured line spread function of the VNIR spectrometer and a spectral response function of the SWIR spectrometer. Due to asymmetric distortions of the LSFs of both sensors and the SRFs of the SWIR sensor a fit with a Gaussian function $G$ would yield distorted estimates of center and resolution. For this reason, the process to retrieve the center and the resolution bandwidth of the response functions is twofold: first, a third-order $B$ spline $F$ is fitted to the measurements to determine the center of a response function as the median $x_{\mathrm{c}}$ of $F$. Then, the resolution $\Delta x$ is centered around $x_{\mathrm{c}}$ and determined by the area under the normalized spline fit $F$, which is equal to the area (0.7610) under a Gaussian function $G(x)$ between its full width half maximum, FWHM. This way, a measure of the response function width is provided in analogy to the full width half maximum of a Gaussian-shaped function. Consequently, the resolution is derived by optimizing the symmetric integration limits $\Delta x / 2$ to satisfy Eq. (24):

$\frac{\int_{x_{\mathrm{c}}-\Delta x / 2}^{x_{\mathrm{c}}+\Delta x / 2} F(x) \mathrm{d} x}{\int_{-\infty}^{\infty} F(x) \mathrm{d} x}=\frac{\int_{-\mathrm{FWHM} / 2}^{\mathrm{FWHM} / 2} G(x) \mathrm{d} x}{\int_{-\infty}^{\infty} G(x) \mathrm{d} x}=0.7610$.

The basic idea to transfer the FWHM concept to asymmetric response functions is also illustrated by the inset in Fig. 16. Using this technique the angular resolution $\Delta \theta$ and the spectral bandwidth $\Delta \lambda$ are determined. In the following, the terms along-track and across-track denote directions perpendicular and parallel to the spatial line respectively.

\subsubsection{Spatial characterization}

Every pixel of the sensor arrays has its own set of LSFs, which are described by the viewing angle $\theta_{\mathrm{c}}$ and the angular resolution $\Delta \theta$. The viewing angles $\theta_{\mathrm{c}}$ of one spatial pixel along the spectral axis are ideally the same. Any deviation therefrom is commonly called keystone. It is defined as the maximum difference between viewing angles for one spatial pixel. The width of LSFs $\Delta \theta$ across- and along-track determines the sharpness of the spatial image.

The geometric and spectral characterizations were done analogous to Gege et al. (2009) and Baumgartner et al. (2012). The measurement setup consists of a narrow slit with a width of $0.05 \mathrm{~mm}$, illuminated by a Quartz Tungsten Halogen lamp and positioned at the focal plane of a reflective
Table 4. Summary of the geometric across-track properties of the specMACS VNIR sensor excluding the first 30 channels.

\begin{tabular}{lrr}
\hline Parameter & Avg. & Min-max \\
\hline Total FOV $\left(^{\circ}\right)$ & 32.7 & - \\
Angular sampling (mrad) & 0.44 & $0.37-0.53$ \\
Angular resolution (mrad) & 1.37 & $0.50-2.89$ \\
Angular resolution (mrad)* & 2.00 & $1.12-2.79$ \\
Angular oversampling & 3.15 & $1.17-5.81$ \\
Keystone (mrad) & 0.30 & $0.06-0.54$ \\
Keystone (pixel) & 0.71 & $0.13-1.23$ \\
\hline
\end{tabular}

* along-track property.

collimator with a focal length of $750 \mathrm{~mm}$. This produces a collimated beam with a divergence of $0.07 \mathrm{mrad}$. A folding mirror directs this beam onto the aperture of the spectrometer. Through linear movement and simultaneous rotation of the folding mirror, different spatial pixels can be illuminated. The collimated beam is large enough to fill the aperture of the spectrometer.

The across-track LSFs are measured by using a slit which is imaged perpendicular to the entrance slit of the spectrograph. The angular scan for the selected pixels is accomplished by changing the illumination angle via the folding mirror over a range of $0.7 \mathrm{rad}$. For the VNIR this scan is done in increments of $0.14 \mathrm{mrad}$ covering the entire FOV. In case of the SWIR instrument, the scan is performed in increments of $0.35 \mathrm{mrad}$.

The along-track LSFs are measured at 7 angles that are evenly distributed over the FOVs of the instruments. They are measured by using a slit that is imaged parallel to the entrance slit of the spectrograph. The incidence angle of the collimated beam on the spectrometer aperture is changed by an along-track translation of the illuminated slit in the focal plane of the collimator. For the measurement of the selected spatial pixels of the VNIR, the along-track LSF is scanned over a range of $6.06 \mathrm{mrad}$ in increments of $0.3 \mathrm{mrad}$, and for the SWIR over a range of $5.9 \mathrm{mrad}$ in increments of $0.15 \mathrm{mrad}$.

To retrieve the viewing angles and angular resolutions from the measurements, the measurements were interpolated using splines as described in Sect. 3.2.2. The geometric along-track values of pixels that are not measured directly are inferred by interpolation of the viewing angles and angular resolution in between the measured spatial pixels. For the interpolation, a second-order polynomial fit to the measured spatial pixel is used. The order of the polynomial functions is selected so that higher order polynomials do not reduce the residuals significantly more. The keystone distortion of one spatial line is defined as the largest difference of across-track viewing angles along the spectral axis.

A typical LSF for the VNIR sensor is shown in Fig. 16a. As previously discussed in Sect. 3.2.2, the LSFs cannot be 


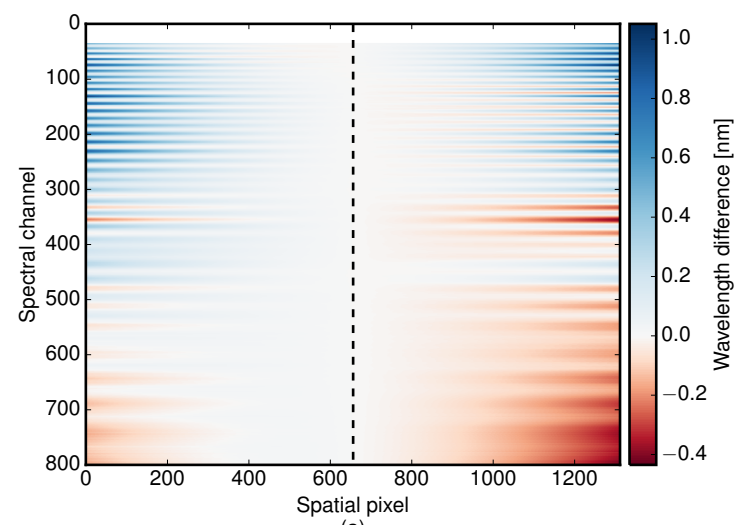

(a)

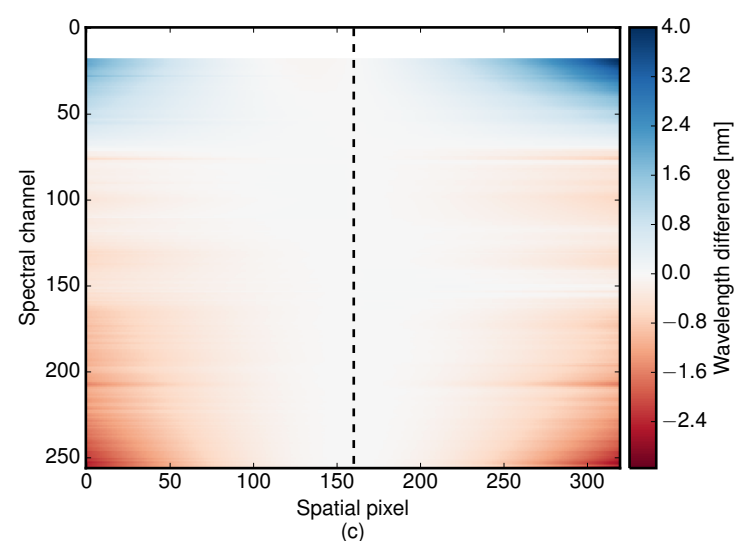

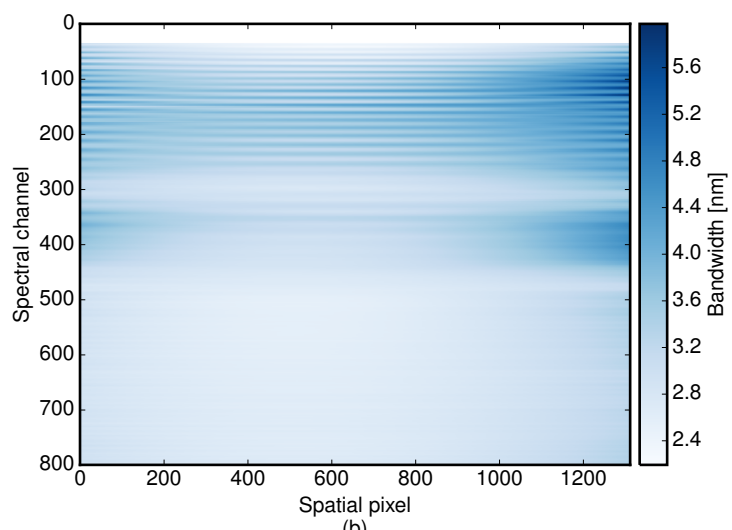

(b)

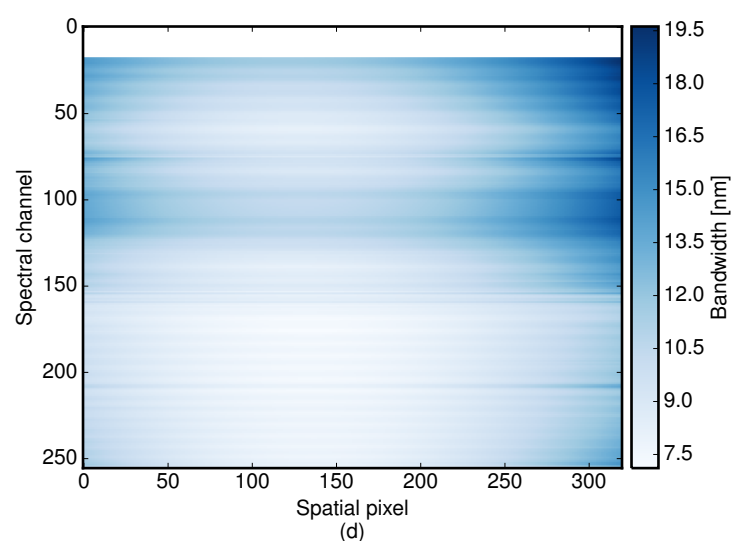

(d)

Figure 18. Spectral characterization results of the VNIR (top) and SWIR (bottom) sensor. Panels (a) and (c) show the difference of the wavelength of each channel to those of pixel 656 (VNIR) and pixel 160 (SWIR) respectively. Panels (b) and (d) show the bandwidth. White areas indicate channels which are excluded from evaluation due to their low sensitivity.

Table 5. Summary of the geometric across-track properties of the specMACS SWIR sensor excluding the first 17 channels.

\begin{tabular}{lrr}
\hline Parameter & Avg. & Min-max \\
\hline Total FOV $\left(^{\circ}\right)$ & 35.5 & - \\
Angular sampling (mrad) & 1.94 & $1.73-2.07$ \\
Angular resolution (mrad) & 3.79 & $2.75-6.60$ \\
Angular resolution (mrad)* & 1.82 & $1.70-2.22$ \\
Angular oversampling & 1.95 & $1.45-3.36$ \\
Keystone (mrad) & 0.50 & $0.27-0.77$ \\
Keystone (pixel) & 0.26 & $0.13-0.41$ \\
\hline
\end{tabular}

* Along-track property.

accurately approximated with Gaussian functions. Therefore, splines were fitted to the measurements to compute the viewing angles $\theta_{\mathrm{c}}$ and the angular resolution $\Delta \theta$ of both spectrometers related to the usual FWHM values for a Gaussianshaped sensitivity. The characterization results for both sensors are shown in Fig. 17 and in Tables 4 and 5. Due to their low sensitivity, some channels of the sensors could not be evaluated accurately. Therefore, the first 30 channels of the
VNIR and the first 17 channels of the SWIR sensor are not taken into account. Figure 17a and $\mathrm{c}$ show the deviations of the across-track viewing angles $\theta_{\mathrm{c}}$ relative to spectral channel 400 for the VNIR and to spectral channel 128 for the SWIR sensor. Ripples in Fig. 17a and b are caused by the etalon effect in the VNIR. For both spectrometer, the strongest keystone distortion occurs at longer wavelengths, while its mean value of $0.30 \mathrm{mrad}$ for the VNIR and $0.50 \mathrm{mrad}$ for the SWIR remain well below the angular resolution of the sensors.

Figure $17 \mathrm{~b}$ and $\mathrm{d}$ show the across-track angular resolution $\Delta \theta$ of the sensors. The area with the smallest $\Delta \theta$ and therefore sharpest pixel is stretched across the sensor FOV. At longer wavelengths and lower pixel numbers, the VNIR image projection gets rather blurry. Besides the achromatism, this behavior could be explained by a slightly misaligned entrance slit with respect to the entrance optics. Considering the mean ratio of 3.15 between angular resolution and sampling and the fact that only parts of the image appear sharp, the VNIR shows a reduced focusability. The sharpest SWIR image projection is approximately at the center in wavelength but asymmetric in lower pixel numbers as it can be seen in Fig. 17d. With a mean angular oversampling of 

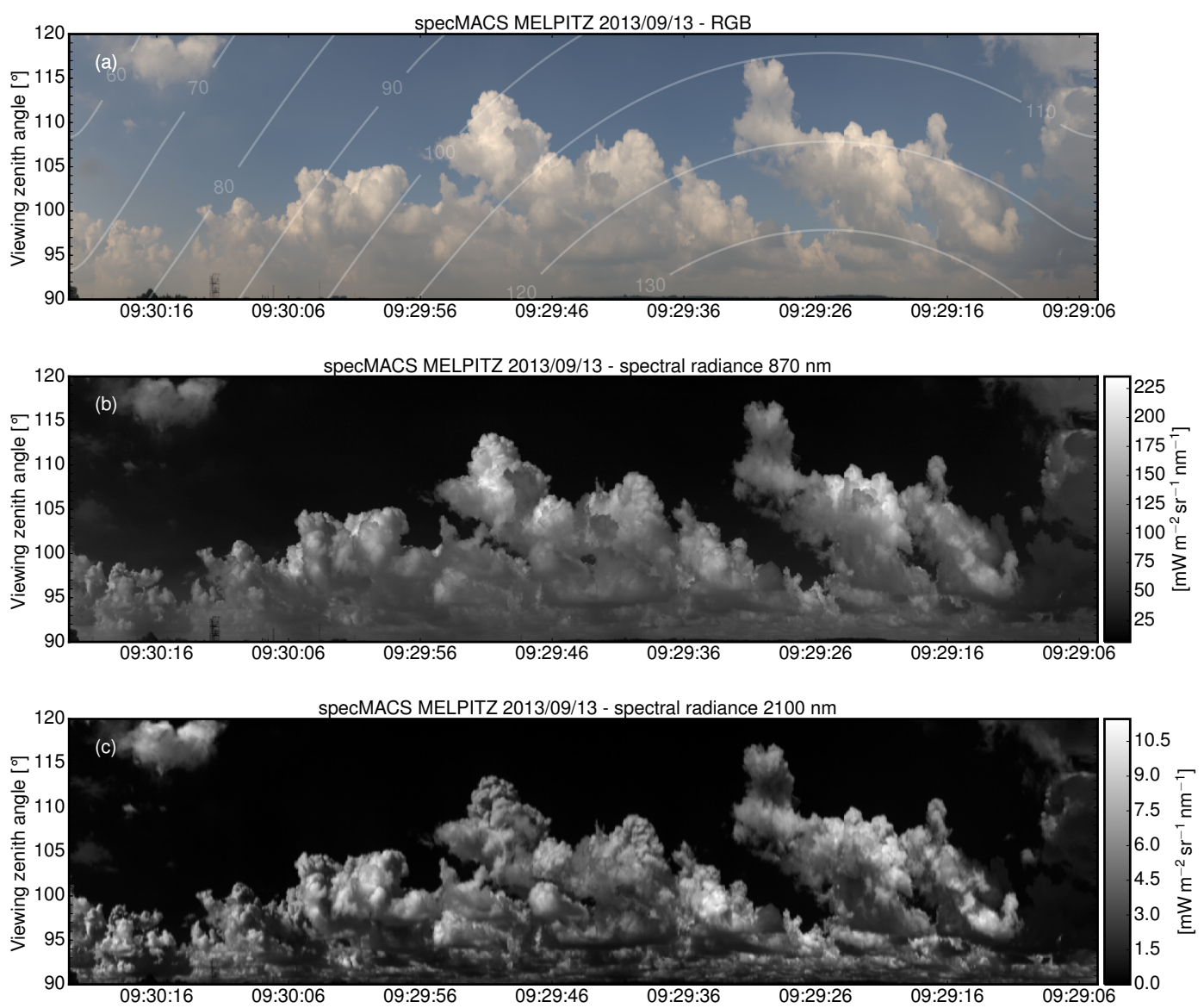

Figure 19. Spectral measurements of cloud sides taken with specMACS on a scanning mount during the HOPE campaign in Melpitz. (a) True-color RGB calculated from the hyperspectral image. (b) Spectral radiance image at $870 \mathrm{~nm}$. (c) Spectral radiance image at $2100 \mathrm{~nm}$. Due to higher absorption and shorter photon path lengths, clouds appear more structured at $2100 \mathrm{~nm}$. The slightly lower radiance from cloud tops at $2100 \mathrm{~nm}$ could be an indication for larger cloud droplets.

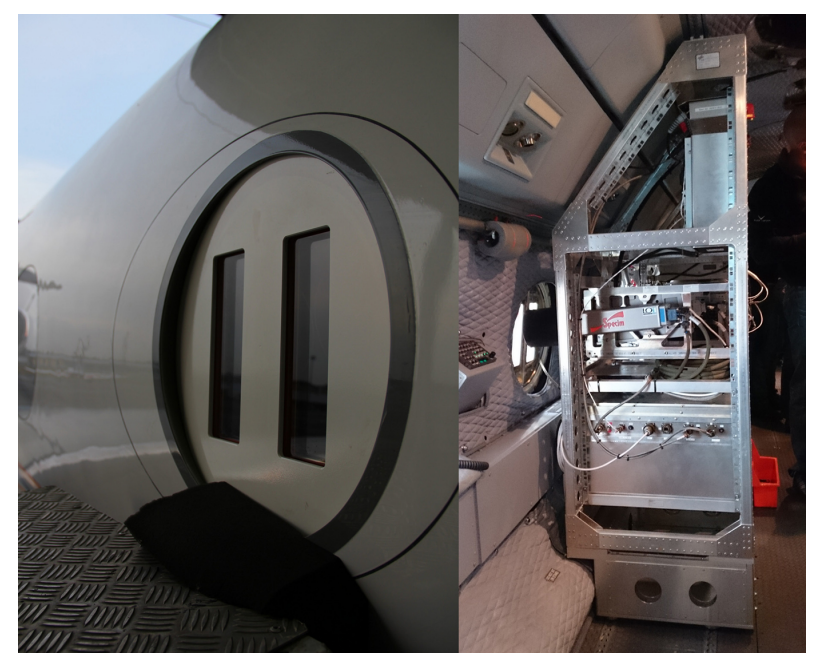

Figure 20. specMACS mounted on the HALO aircraft equipped with the side view port.
1.95, the SWIR optics produce a sharper image on the detector. However, the angular sampling $(1.94 \mathrm{mrad})$ and resolution $(3.79 \mathrm{mrad})$ are far coarser than for the VNIR (0.44 and $1.37 \mathrm{mrad})$.

The deviations in along-track viewing angles $\theta_{\mathrm{c}}$ and alongtrack angular resolutions $\Delta \theta$ are not shown here since their values are similar to their across-track values. Both alongtrack properties exhibit an even more symmetrical distribution over the sensors.

\subsubsection{Spectral characterization}

For one spectral channel, the SRF center and its width can vary over the FOV of the instrument, i.e. every single pixel of the sensor array has its individual SRF, similar to the LSFs. The deviation of the center wavelength $\lambda_{\mathrm{c}}$ within a spatial line is commonly described as spectral smile while the SRF width gives the spectral bandwidth $\Delta \lambda$. In the following the spectral smile will be given as the deviation of $\lambda_{\mathrm{c}}$ with respect to the center pixel within each spatial line. 


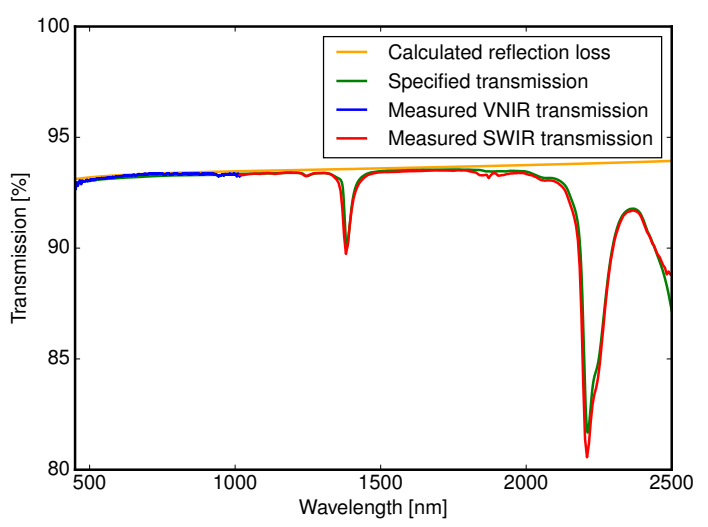

Figure 21. Spectral transmission and calculated reflection loss of the HALO side view port.

To measure the SRF, a collimated beam of nearly monochromatic light from a monochromator is used. The previously discussed folding mirror directs the collimated beam onto the aperture of the spectrometer. This allows a single spatial pixel to be selected for illumination. A detailed sketch of the calibration setup can be found in Gege et al. (2009) in Fig. 7. To guarantee that the spectrometer aperture and IFOV are completely illuminated, the beam cross section is larger than the aperture and the beam divergence is larger than the IFOV of the spectrometer. The monochromator has an absolute uncertainty of $\pm 0.1 \mathrm{~nm}$ for wavelengths below $1000 \mathrm{~nm}$ and $\pm 0.2 \mathrm{~nm}$ for longer wavelengths. The spectral bandwidth is set to $0.65 \mathrm{~nm}$ for the measurement of the VNIR and $1.3 \mathrm{~nm}$ for the measurement of the SWIR. Computations indicate that the chosen bandwidth of the monochromator has only very little influence on the measured bandwidths as long as the monochromator bandwidth is smaller than the measured bandwidth and its SRF is known. Both requirements are met with a Gaussian monochromator SRF well below the specified spectrometer bandwidth. For the measurement of the SRFs of the VNIR, the wavelength of the monochromator is scanned from 400 to $1030 \mathrm{~nm}$ in steps of $1 \mathrm{~nm}$, and for the SWIR, from 940 to $2550 \mathrm{~nm}$ in steps of $2 \mathrm{~nm}$. Due to time constraints, these measurements are only feasible for a small subset of all spatial pixels. For both sensors, the SRFs are measured at seven angles evenly distributed over their across-track FOV.

The spectral properties of the other pixels are inferred by fitting the center wavelengths and bandwidths with a secondorder polynomial. This procedure assumes that the properties of the optical system do not vary rapidly on the scale of the detector array. This assumption holds for the specMACS imaging spectrometers, which was validated using spectral line lamps. The spectral smile for each spatial pixel is computed as the difference between its wavelength and the wavelength of the center pixel within the same spectral channel.

The measurement setup is described in more detail in Gege et al. (2009) and details about the data analysis as well as a

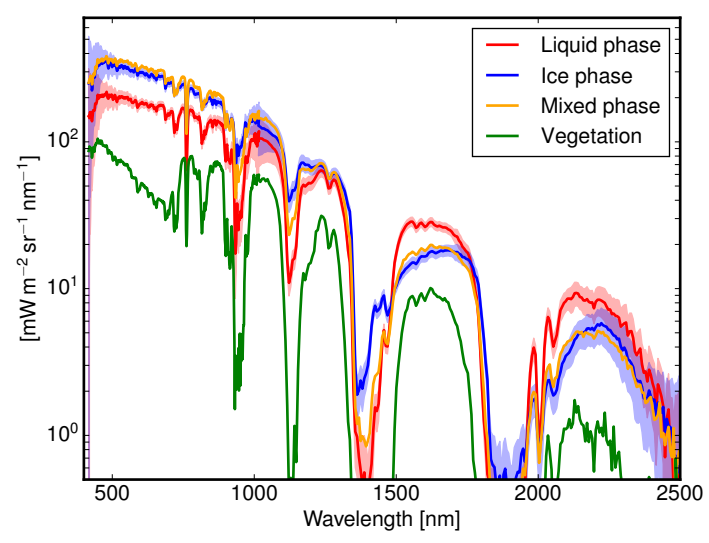

Figure 22. Reflected solar spectra measured with specMACS onboard HALO during the ACRIDICON-CHUVA campaign in Brazil.

validation of the approach for another hyperspectral camera can be found in Baumgartner et al. (2012).

A measured SRF of the SWIR sensor can be seen in Fig. 16b. The figure shows an asymmetric response with a second peak at shorter wavelengths. The results of the spectral characterization can be seen in Fig. 18 and Tables 6 and 7. Just like during the geometric characterization some channels are not evaluated. The first 36 channels of the VNIR and the first 17 channels of the SWIR sensor are skipped due to low sensitivity in these regions.

Figure 18a and c illustrate the smile distortion. For the VNIR sensor, the magnitude of the average spectral smile is between 0.1 and $1.1 \mathrm{~nm}$. For the SWIR sensor, the magnitude of the average spectral smile is on the order of $1.1 \mathrm{~nm}$, ranging from 0.1 to $4.1 \mathrm{~nm}$. Note that the sign of the smile curve changes between the bottom half and the top half of both detector arrays.

Figure $18 \mathrm{~b}$ and $\mathrm{d}$ show the spectral bandwidth of each detector element. It is about $3.1 \mathrm{~nm}$ in average for the VNIR sensor, and degrades to $6.0 \mathrm{~nm}$ at the spatial edges of the detector array. For the SWIR sensor, spectral bandwidth is about $10.3 \mathrm{~nm}$ at the center of the detector array and increases up to $19.6 \mathrm{~nm}$ at the spatial edges of the array. For the VNIR, spectral oversampling is 4.03 . This allows the spectral sampling to be reduced by half without losing information. In contrast, the average SWIR spectral oversampling is only around 1.64 .

The ripple features in the plots of Fig. 18a and b are caused by the etalon effect.

\subsubsection{Optical distortion correction}

Optical distortion correction can be performed through interpolation of the data set onto a regular grid. As the adequate grid depends on the particular application, and as interpolation for every pixel is lossy in terms of information content, this interpolation step should be performed during spatial rectification of the image; hereby the optical charac- 
Table 6. Summary of the spectral properties of the specMACS VNIR sensor excluding the first 36 channels.

\begin{tabular}{lrl}
\hline Parameter & Avg. & Min-max \\
\hline Spectral range (nm) & - & $421.3-1017.5$ \\
Spectral sampling (nm) & 0.8 & $0.6-1.0$ \\
Spectral bandwidth (nm) & 3.1 & $2.2-6.0$ \\
Spectral oversampling & 4.03 & $3.08-7.82$ \\
Spectral smile (nm) & 0.3 & $0.1-1.1$ \\
Spectral smile (pixel) & 0.38 & $0.07-1.40$ \\
\hline
\end{tabular}

Table 7. Summary of the spectral properties of the specMACS SWIR sensor excluding the first 18 channels.

\begin{tabular}{lrl}
\hline Parameter & Avg. & Min-max \\
\hline Spectral range (nm) & - & $1017.8-2505.5$ \\
Spectral sampling (nm) & 6.3 & $5.2-6.9$ \\
Spectral bandwidth (nm) & 10.3 & $7.1-19.6$ \\
Spectral oversampling & 1.64 & $1.15-3.10$ \\
Spectral smile (nm) & 1.1 & $0.1-4.1$ \\
Spectral smile (pixel) & 0.18 & $0.02-0.65$ \\
\hline
\end{tabular}

terization for each instrument is combined with the location information for each frame. This is then stored together with the radiometric signal from each pixel. Subsequently, these metadata can be used in the transformation onto the final target coordinate system.

\section{Ground-based and airborne applications}

First deployments of the specMACS instrument were the ground-based measurement campaign HOPE in Melpitz, Germany, in September 2013 and the aircraft campaign ACRIDICON-CHUVA in the Amazon region around Manaus, Brazil, in September 2014 (Wendisch et al., 2016). While specMACS was put on a scanning mount during the ground-based campaign, the instrument was installed on the German research aircraft HALO (High Altitude LOng range aircraft, Krautstrunk and Giez, 2012) during the ACRIDICON-CHUVA campaign.

\subsection{Ground-based setup}

For ground-based measurements, specMACS is mounted on a scanning mount (MESU-OPTICS Mesu-Mount 200) with two rotating axes (vertical and horizontal). This allows the instrument to be pointed anywhere in the upper hemisphere and to perform precise and repeatable automated scans. The scanning mount is equipped with a customized closed loop motor controller (based on an ROBOTEQ Inc., SDC2130). It is equipped with an optical relative position encoder. This allows the full turn of each axis to be controlled in 10000 steps, which results in a relative positioning accuracy of $0.072^{\circ}$. This accuracy is comparable to the IFOV of the sensors.

\subsubsection{Ground-based measurements}

An exemplary data set, measured during the ground-based campaign, is given in Fig. 19. The first panel (Fig. 19a) shows a true-color image that was rendered using spectral radiance data from the VNIR camera. Here, corresponding scattering angles towards the sun are shown as isolines. The next two panels show calibrated radiances for the same scene as they were measured with the VNIR spectrometer at $870 \mathrm{~nm}$ (Fig. 19b) and with the SWIR spectrometer at $2100 \mathrm{~nm}$ (Fig. 19c). The more structured appearance of clouds at $2100 \mathrm{~nm}$ can be attributed to shorter photon path lengths due to a higher absorption by cloud droplets at this wavelength. With longer photon path lengths, the transport of photons between adjacent cloud regions becomes important. This leads to radiative smoothing of radiances from clouds at non-absorbing wavelengths. Furthermore, the slightly lower radiance from cloud tops at $2100 \mathrm{~nm}$ could be an indication for larger cloud droplets. This new perspective on clouds is an essential step towards the proposed microphysical retrievals from cloud sides (Zinner et al., 2008; Martins et al., 2011), since up to now, most imaging spectrograph instruments were designed for the nadir-looking perspective.

\subsection{Airborne setup}

For airborne measurements, specMACS was mounted into a HALO Rack facing sideways with vertical spatial axis in cooperation with enviscope GmbH. For this task, a specifically designed window for the HALO side view port had to be developed to ensure a high transmissivity over the whole spectral range of specMACS (Fig. 20). Two purified quartz glass panes (Herasil 102, Haereus) $2 \mathrm{~cm}$ thick were embedded into two vertical apertures inside the side view port. To address the problem of window icing, a fan was installed below the window, which constantly blows warm cabin air onto the inner window surface.

The cameras' field of view was tilted $5^{\circ}$ downward about the longitudinal axis of the airplane. After matching the field of view of both cameras, a combined field of view remains available between $21^{\circ}$ below and $11^{\circ}$ above the horizon for level flight.

During the airborne operation the across-track pixel size for clouds in a distance of $5 \mathrm{~km}$ is around $2.2 \mathrm{~m}$ for the VNIR and $9.7 \mathrm{~m}$ for the SWIR in accordance with their respective angular sampling. In order to obtain a comparable spatial along-track resolution, the frame rate is set to $30 \mathrm{fps}$. With a maximum ground speed of $800 \mathrm{~km} \mathrm{~h}^{-1}$ the pixel size for clouds in this distance becomes $2.2 \times 7.4 \mathrm{~m}$ for the VNIR and $9.7 \times 7.4 \mathrm{~m}$ for the SWIR. Internal storage was designed large enough to enable continuous measurements for at least two flights of $8 \mathrm{~h}$ duration. 

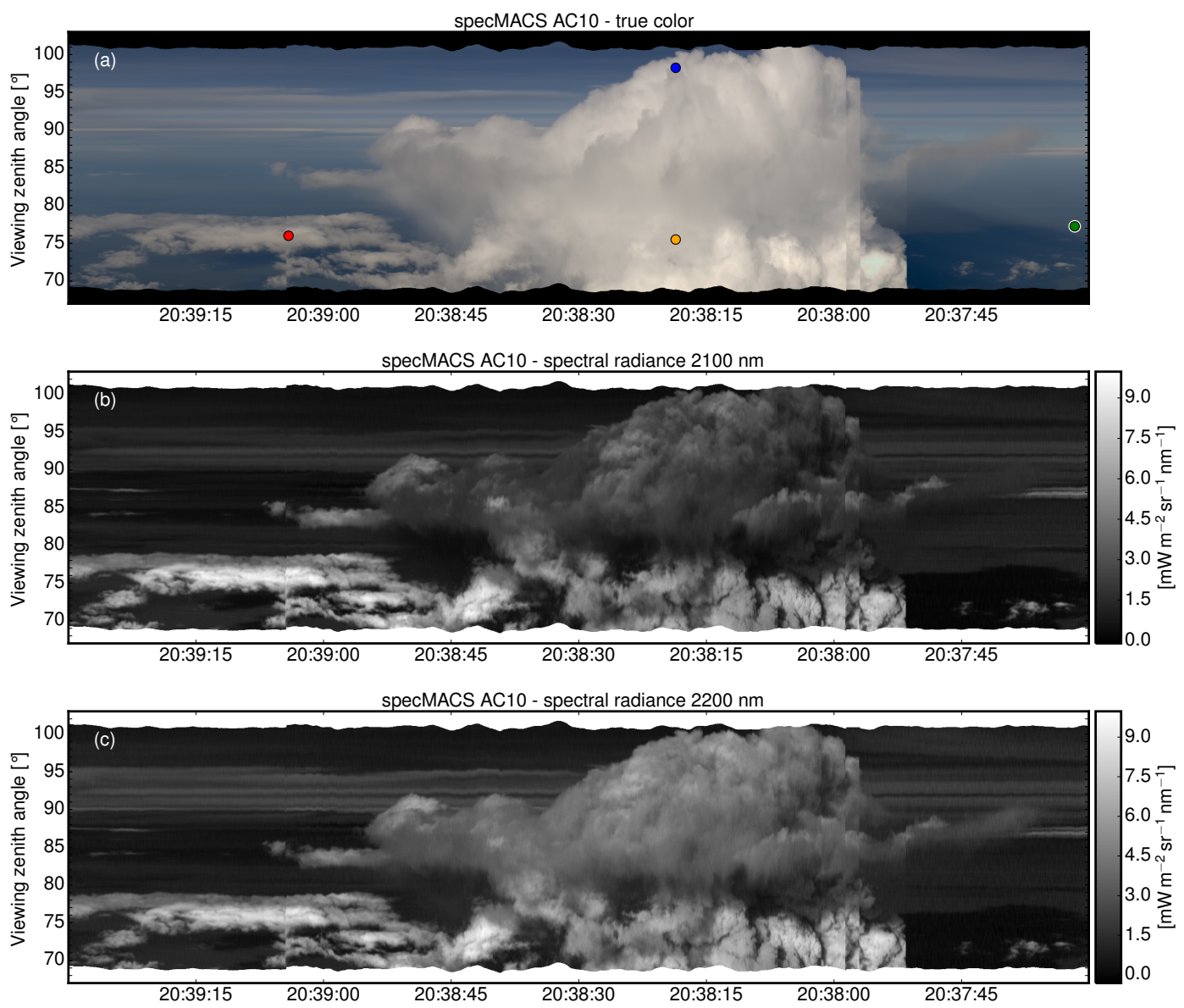

Figure 23. Spectral measurements of cloud sides taken with specMACS onboard HALO during the ACRIDICON-CHUVA campaign in Brazil. (a) True-color RGB calculated from the hyperspectral image, (b) spectral radiance image at $2100 \mathrm{~nm}$, (c) spectral radiance image at $2200 \mathrm{~nm}$. The ice phase is clearly visible as a distinct drop of the spectral radiances from panel (c) at $2200 \mathrm{~nm}$ to panel (b) at $2100 \mathrm{~nm}$. The drop in radiance can be explained by the stronger ice absorption coefficient at $2100 \mathrm{~nm}$ compared to $2200 \mathrm{~nm}$.

Since the instrument was fixed in one of the measurement racks of HALO, the captured data have to be rectified during post-processing to correct for airplane movements. The spatial rectification can be done using inertial navigation systems (INSs) provided by the BAsic HALO Measurement And Sensor System (BAHAMAS; Krautstrunk and Giez, 2012) or by the Spectral Modular Airborne Radiation measurement sysTem (SMART; Wendisch et al., 2001), whose subsystems offer both a $100 \mathrm{~Hz}$ data stream of accurate position information.

\subsubsection{Window transmission}

The transmission of the quartz glass windows was characterized radiometrically and spectrally on the CHB large integrating sphere by comparing specMACS measurements of the sphere with and without the windows in the optical path. The angular dependence of the window transmission was further characterized at three different angles between the optical axis of the sensors and the window $\left(0^{\circ}=\right.$ perpendicular to the optical axis, $11.8^{\circ}=$ angle as mounted on HALO, $15.5^{\circ}=$ steepest angle possible with the chosen experimental setup). Note that the transmission,

$T=\frac{L_{\text {Win }}}{L}=\frac{R^{-1} s_{\mathrm{n} ; \mathrm{Win}}}{R^{-1} s_{\mathrm{n}}}=\frac{s_{\mathrm{n} ; \mathrm{Win}}}{s_{\mathrm{n}}}$,

can be calculated based on the dark-current- and nonlinearity-corrected signals alone and without using the characterization of the absolute radiometric response. In addition to the laboratory characterization and transmission values as specified by the manufacturer, theoretical reflection losses of the window surface including internal reflections were calculated using refractive indices from the glass data sheet (varying from 1.4703 to 1.4280 in our wavelength range) and Snell's and Fresnel's laws for comparison.

The spectral transmission of the side view port is shown in Fig. 21. As expected, the theoretical reflection loss calculation yields an upper estimate for the actual transmission because of the missing absorption. The low discrepancy between specification and measurement and the close 
match of the overlapping region between both sensors show the high relative accuracy of the sensors and indicate that the nonlinearity correction works as intended. The two absorption bands in the spectrum show the expected strong IRabsorption of remaining $\mathrm{OH}$ species in fused quartz glass.

Significant spatial variation of the window transmission has not been observed, however, small reflections of the sensors optical systems with an intensity of up to $0.5 \%$ of the direct transmission were found.

\subsubsection{Airborne measurements}

In Fig. 22 examples of reflected solar spectra are shown which were measured with the specMACS instrument on flight AC10 (12 September 2014) during the ACRIDICONCHUVA campaign. The shaded regions show the overall radiometric $2 \sigma$ uncertainty which was estimated using Eq. (23); hereby, a fully polarized signal $(p=1)$ was assumed to obtain an upper estimate of the radiometric uncertainty. At the edges of the spectra, at the transition between VNIR and SWIR around $1000 \mathrm{~nm}$ and within the water vapor absorption bands, the overall radiometric uncertainty reaches values of up to $50 \%$ due to low signal levels. Around 1.3, 1.6, and $2.1 \mu \mathrm{m}$, the radiometric uncertainty remains below $10 \%$ for well-illuminated scenes. In the visible and near-infrared spectral range, the error even remains well below $5 \%$.

The locations of the spectra shown in Fig. 22 are indicated in Fig. 23 by points with corresponding color. While below $1000 \mathrm{~nm}$ the spectral radiance from the ice cloud (blue line) is higher than that from the liquid water cloud (red line), the spectral radiance from the liquid water cloud is higher at longer wavelengths. The lower radiance of the ice cloud at longer wavelengths can be explained by the higher absorption coefficient of ice and with the usually larger size of ice particles. Due to a higher absorption, the ice cloud phase can also be distinguished from the liquid cloud phase by their different spectral slope between 1500 and $1700 \mathrm{~nm}$ (Ehrlich et al., 2008) and 2100 and $2200 \mathrm{~nm}$ (Martins et al., 2011). With a spectral slope in between the ice and liquid phase, the spectrum of a cloud region with mixed phase is shown in orange. Due to the spectral signature of chlorophyll, the near-infrared edge of vegetation on the ground (green line) is easy to recognize as a distinct jump in radiance between 680 and $730 \mathrm{~nm}$.

Figure 23a shows the true-color image corresponding to Fig. 22 that was rendered using spectral radiance data from the VNIR camera. Calibrated radiances at 2200 and $2100 \mathrm{~nm}$ are shown below in Fig. 23b and c. Since the ice absorption is stronger at $2100 \mathrm{~nm}$ compared to $2200 \mathrm{~nm}$, the cloud ice phase becomes visible as an evident drop in radiance at $2200 \mathrm{~nm}$.

Corresponding to the shaded regions in Fig. 22, Fig. 24 shows the spatial distribution of the overall radiometric $2 \sigma$ uncertainty for the same scene at the near-infrared wavelength $870 \mathrm{~nm}$ (Fig. 24a) and the shortwave infrared wave- length $2100 \mathrm{~nm}$ (Fig. 24b). At $870 \mathrm{~nm}$, the radiometric error is very low $(<5 \%)$ for well-illuminated clouds and ground regions. Shaded ground and clear-sky regions exhibit larger radiometric uncertainty of up to $10 \%$. Due to a lower sensor sensitivity, the same radiometric uncertainty is given for well-illuminated cloud scenes at $2100 \mathrm{~nm}$. Here, radiances from shaded cloud and ground regions can only be determined with a very large uncertainty of $20 \%$ or more. In the SWIR spectral range, the limiting factor to radiometric accuracy is the unknown dark signal drift between dark frame measurements.

\section{Conclusions and outlook}

The hardware design and the modular and resilient software design enables the specMACS system to be used as a versatile data acquisition system for hyperspectral measurements in the wavelength range of 417 to $2496 \mathrm{~nm}$. The design can easily be adapted to ground-based and airborne measurements and can be extended to or combined with even more sensors naturally (like a long-wave infrared camera). The software concept proved to be reliable and facilitated measurements throughout the whole ACRIDICON 2014 campaign autonomously and without any measurement interruptions.

The laboratory characterization of the VNIR and SWIR sensors revealed important details of the behavior of the sensors needed for a scientific application of specMACS. Of particular value is the characterization of the previously unknown nonlinear behavior of the VNIR. The nonlinearity correction provides a consistent calibration of both sensors. This allows to merge the spectra of both sensors to a single VNIR/SWIR spectrum.

The available error budget calculation now allows to estimate the significance of different radiometric uncertainties. For the VNIR, major contributions to the overall radiometric uncertainty of around $5 \%$ are caused by the calibration uncertainty of $R$ (error of $\approx 3 \%$ ) and the polarization sensitivity for highly polarized light (error $\leq 5 \%$ for fully polarized light). Without the nonlinearity correction, the radiometric signal would furthermore be strongly biased ( $-9 \%$ at high signal levels). For the SWIR, major error contributions to the overall radiometric uncertainty of around $10 \%$ are caused by the uncertainty of the absolute radiometric standard itself (error of 5 to $10 \%, \lambda>1700 \mathrm{~nm}$ ) and the dark signal drift for low exposed regions (error of $20 \%$ and more, depending on the frequency of dark frame measurements).

However, there are several points which have not been considered during the described effort to characterize the instrument thoroughly. Without claiming completeness, the following effects might be worth investigating further.

1. Dark signal variability has only partially been explored in a controlled fashion. Since dark signals are measured frequently in the described setup, variations are directly 

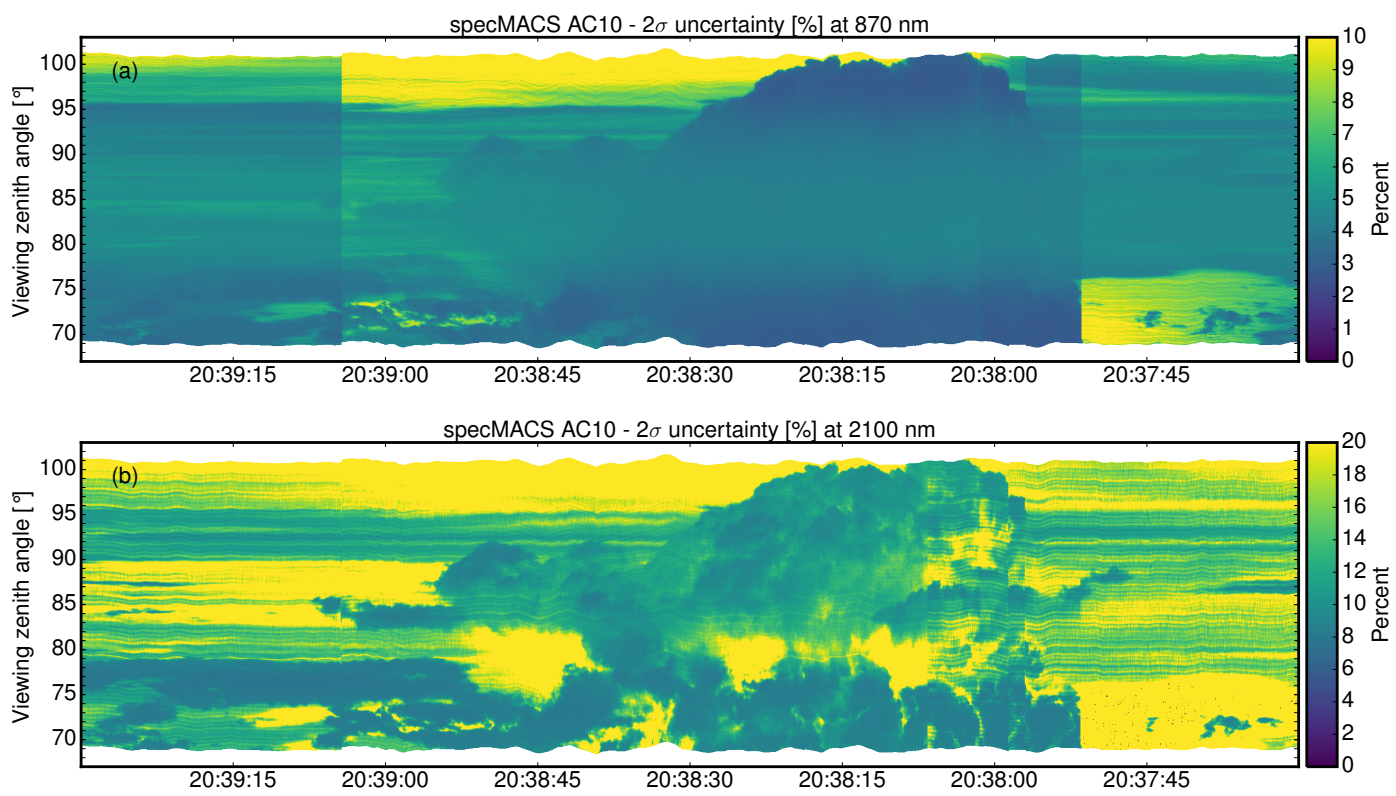

Figure 24. Relative uncertainties $(2 \sigma)$ in percent for the spectral measurements of cloud sides shown in Fig. 23 (a) at $870 \mathrm{~nm}$ and (b) at $2100 \mathrm{~nm}$.

considered and do not need to be characterized. If future applications change the measurement mode, for which timely dark measurements are not possible, a more indepth characterization would be needed.

2. The dark signal behavior for very large temperature swings has not been thoroughly investigated. Frequent dark frame measurements and the avoidance of direct sunlight onto the instrument are therefore essential during outside ground-based measurements.

3. The radiometric response $R$, including FPN, might change over time and environment conditions (e.g., temperature). Reliable statements about the long-term calibration stability can only be made in subsequent calibration efforts in the future.

4. Due to the difficulty of establishing a bright light source with spectrally stable and precisely linearly adjustable intensity, the radiometric nonlinearity has not been investigated directly in terms of incoming radiance alone. A deeper investigation of this behavior might show additional nonlinearity effects. There is some indication that these additional effects might not be dominant, as suggested in Sect. 3.1.2.

5. The effectiveness of the final stray light protection has only been simulated and subjectively assessed. A dedicated characterization would yield final evidence for the effectiveness.

Despite these open issues, the overall radiometric uncertainty estimation can be relied on, if the following points are considered during the measurement with specMACS and during the subsequent calibration of scientific data.

1. For both instruments, no serious internal stray light and ghost images have been found. When direct sunlight impinges on the front optics, stray light baffles become indispensable.

2. Due to the variable dark current level of the SWIR sensor prompt and frequent dark signal measurements for every used integration time and sensor temperature are essential to achieve the specified radiometric accuracy. Interpolation of dark signal frames from before and after each measurement are needed to compensate for the SWIR dark signal drift ( $\leq 30 \mathrm{DN}$ per minute). In contrast, the VNIR dark signal shows no strong dependence on integration time or sensor temperature since it is mainly caused by read-out noise.

3. The radiometric response $R$ given from the manufacturer does not differ by more than $10 \%$ from $R$ found in this work. Although $R$ seems to be quite stable, the calibration should be repeated over time since the radiometric uncertainty is about $3 \%$ in the best wavelength region.

4. For the SWIR, we have found a small mismatch between the integration time set $t_{\text {set }}$ and the actual integration time $t_{\text {int }}$. For this reason, we introduced an additional term $t_{\text {ofs }}$ to compensate for this mismatch.

5. The radiometric response of the VNIR shows nonlinear behavior at medium to large signal levels, which leads 
to an underestimation of the absolute radiometric signal if not corrected.

6. During the spatial characterization, the VNIR sharpness turned out to be suboptimal. Besides a slight achromatism, the focus seems to shift in the across-track direction with wavelength.

7. The spectral bandwidth is within specifications for both spectrometers. The spectral sampling is sufficient for both instruments, while the oversampling of the VNIR spectrometer allows the reduction of the spectral sampling by half without losing information significantly.

8. During the spectral and spatial characterization, no significant spectral smile or keystone was found for both cameras.

9. Both sensors exhibit a certain polarization sensitivity, which for the most part remains well below $5 \%$. In the worst case of completely polarized light with unknown polarization orientation, this results in an additional radiometric uncertainty of $5.3 \%$.

The final evaluation shows that the instrument performance complies with the accuracy requirements stated in the introduction. Absolute radiometric accuracy well below the mentioned 3-D radiative effects can be achieved when the described signal calibration procedure is applied. The radiometric error budget proves that the radiometric uncertainty for well-illuminated cloud scenes can be kept well below $20 \%$ over the full wavelength range of the instrument. This is also confirmed by the good agreement between both spectrometers in the overlap region around $1000 \mathrm{~nm}$. As demanded in Sect. 1.2, the spectral bandwidth is the limiting factor for the spectral accuracy of the instrument. More precisely, the spectral bandwidth of the VNIR with $3.1 \mathrm{~nm}$ is well above the calculated spectral smile of $0.3 \mathrm{~nm}$ and 1 order of magnitude larger than the spectral calibration accuracy of $\pm 0.1 \mathrm{~nm}$. Additionally, the SWIR spectral bandwidth of $10.3 \mathrm{~nm}$ is larger by 1 order of magnitude than its spectral smile of $1.1 \mathrm{~nm}$ and larger by 2 orders of magnitude compared to the spectral calibration accuracy of $\pm 0.2 \mathrm{~nm}$. Spectral calibration accuracy fully meets the requirements of current microphysical cloud retrievals and enables reliable identification of gaseous absorption lines. The spectral bandwidth below $1000 \mathrm{~nm}$ should be sufficient for the analysis of absorption band depths of features like the oxygen $A$ band.

As shown in Sect. 4, measurements acquired during the ACRIDICON 2014 campaign offer many possibilities for data analysis. The in situ data simultaneously acquired by other participating institutions yield a unique opportunity to validate retrieved remote sensing results with directly measured cloud properties. 


\section{Appendix A: Instrument automation}

\section{A1 Auto exposure}

The main task of the auto-exposure control system, setting the integration time $t_{\text {int }}$ to an optimized value, was designed with three goals of descending importance in mind. Since clouds as the main object of interest are typically the brightest parts of a scene, overexposure is to be avoided in any case. To limit the number of distinct dark current measurements and to facilitate later data analysis, only a few discrete integration times will be used. These are indicated as $t_{\text {int }}(i)$ in the following. However, to recover from very bright scenes and to use the available dynamic range of the sensor to a full extent, integration time should be increased after a certain time span of underexposed conditions.

In Fig. A1 the overall logic of the integration time regulation of the auto-exposure software is illustrated. The logic is based on a histogram of the signal which is evaluated in real time over all spatial and spectral pixels. From the histogram, the 99th percentile $\left(q_{99}\right)$ is calculated and stored for subsequent analysis. The $q_{99}$ was chosen since it turned out to be a more stable indicator for current signal levels than the maximum value, which is sensitive to signal noise and bad pixels.

A limited set of integration times $t_{\text {int }}(i)$ were used during the aircraft measurement campaign ACRIDICON 2014: 0.5, $0.85,1,1.2,1.5,2,3,5,8,12,18$, and $25 \mathrm{~ms}$. These values were chosen as a compromise between a sufficient range of values, reasonably small steps (less than a factor of 2) between integration times, and the goal to have only a limited number of distinct integration times.

The following algorithm is in principle independent of the frame rate, but was tested and optimized for $30 \mathrm{fps}$. To avoid overexposure, the 99th percentile $q_{99}$ of the signal histogram is limited to $3 / 4$ of the full dynamic range of the sensor in order to provide headroom for transient radiance peaks. If this limit is exceeded for more than four frames within the last 150 frames $(5 \mathrm{~s} @ 30 \mathrm{fps})$, the integration time $t_{\text {int }}(i)$ is reduced to the next allowed value $t_{\text {int }}(i-1)$. After such an overexposure protection is triggered, no increments to longer integration times are allowed during the following 1800 frames (1 min@30fps).

To recover from a reduced integration time, the autoexposure control periodically tries to increase the integration time $t_{\text {int. }}$. To this end, the histograms of the last 150 frames are periodically (e.g., every $30 \mathrm{~s}$ ) extrapolated to the next longer integration time $t_{\text {int }}(i+1)$. If the extrapolated histograms do not trigger the overexposure protection described above, $t_{\text {int }}(i)$ is increased to $t_{\text {int }}(i+1)$. Thereby, any increase of integration time is tested before it is actually performed and suppressed if the signal limit set by the quantile limit were exceeded.

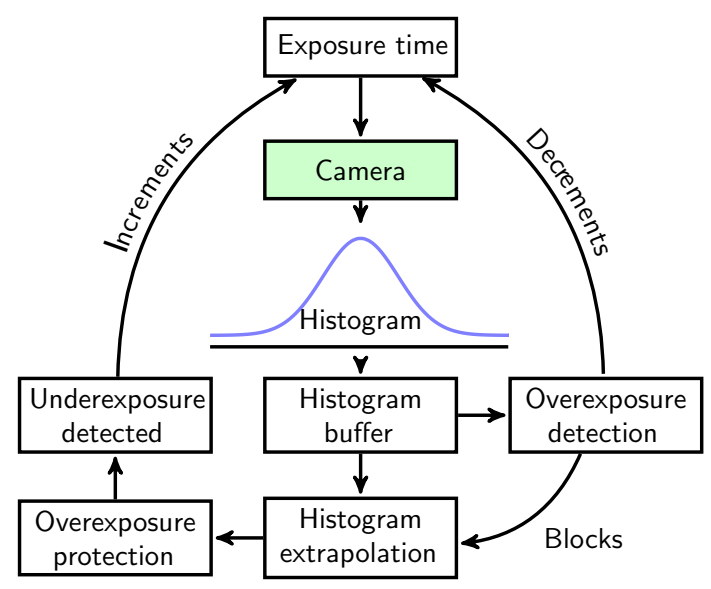

Figure A1. Overview of the automated exposure control system.

\section{A1.1 Automatic dark frame}

Another task of the control software is the automation of dark signal measurements. During dark signal measurements, it is obviously not possible to perform real measurements, so the amount of time spent on dark signal measurements is to be minimized. However, the dark signal varies with time, so the automation is set up to measure approximately 30 dark frames at least every $2 \mathrm{~min}$. Since the dark signal additionally changes with integration time $t_{\text {int }}(i)$, the system checks if a recent dark signal measurement with the current sensor settings was obtained and if not, triggers a dark signal measurement before changing $t_{\mathrm{int}}(i)$. 
Acknowledgements. We thank Meinhard Seefeldner and Anton Lex for their great support in constructing and building the stray light protection and many other mechanical parts for the specMACS system. Florian Ewald gratefully acknowledges funding of parts of this work by the German Research Foundation (DFG) under grant number MA 2548/9-1. We thank Karim Lenhard and Peter Gege (IMF) for their support during the laboratory characterization. We would also like to thank enviscope GmbH (D. Schell), Uni Leipzig (M. Wendisch), Max Planck Institute for Chemistry (U. Pöschl) and the DLR Institute of Atmospheric Physics (M. Rapp) for their support in adapting and operating specMACS on HALO.

Edited by: S. Schmidt

\section{References}

Aikio, M.: Hyperspectral prism-grating-prism imaging spectrograph, Technical Research Centre of Finland Heinola, Finland, 36-78, 2001.

Armstrong, J.: Programming Erlang, The Pragmatic Bookshelf, Raleigh, NC and Dallas, TX, USA, 1-383, 2007.

Babey, S. and Anger, C.: A compact airborne spectrographic imager (CASI), in: Quantitative remote sensing: An economic tool for the Nineties, 2, 1028-1031, 1989.

Baumgartner, A.: Characterization of Integrating Sphere Homogeneity with an Uncalibrated Imaging Spectrometer, in: Proc. WHISPERS 2013, 1-4, 2013.

Baumgartner, A., Gege, P., Köhler, C., Lenhard, K., and Schwarzmaier, T.: Characterisation methods for the hyperspectral sensor HySpex at DLR's calibration home base, in: Sensors, Systems, and Next-Generation Satellites XVI, vol. 85331H, doi:10.1117/12.974664, 2012.

Bierwirth, E., Ehrlich, A., Wendisch, M., Gayet, J.-F., Gourbeyre, C., Dupuy, R., Herber, A., Neuber, R., and Lampert, A.: Optical thickness and effective radius of Arctic boundary-layer clouds retrieved from airborne nadir and imaging spectrometry, Atmos. Meas. Tech., 6, 1189-1200, doi:10.5194/amt-6-1189-2013, 2013.

Bohndiek, S. E., Blue, A., Clark, A., Prydderch, M., Turchetta, R., Royle, G., and Speller, R.: Comparison of Methods for Estimating the Conversion Gain of CMOS Active Pixel Sensors, IEEE Sens. J., 8, 1734-1744, doi:10.1109/JSEN.2008.2004296, 2008.

Bragg, W. H. and Bragg, W. L.: The Reflection of X-rays by Crystals, P. Roy. Soc. A, 88, 428-438, doi:10.1098/rspa.1913.0040, 1913.

Bréon, F. and Doutriaux-Boucher, M.: A comparison of cloud droplet radii measured from space, IEEE T. Geosci. Remote, 43, 1796-1805, 2005.

Chiu, J. C., Marshak, A., Huang, C.-H., Várnai, T., Hogan, R. J., Giles, D. M., Holben, B. N., O'Connor, E. J., Knyazikhin, Y., and Wiscombe, W. J.: Cloud droplet size and liquid water path retrievals from zenith radiance measurements: examples from the Atmospheric Radiation Measurement Program and the Aerosol Robotic Network, Atmos. Chem. Phys., 12, 1031310329, doi:10.5194/acp-12-10313-2012, 2012.

Coddington, O. M., Pilewskie, P., Redemann, J., Platnick, S., Russell, P. B., Schmidt, K. S., Gore, W. J., Livingston, J., Wind, G., and Vukicevic, T.: Examining the impact of over- lying aerosols on the retrieval of cloud optical properties from passive remote sensing, J. Geophys. Res., 115, D10211, doi:10.1029/2009JD012829, 2010.

Coddington, O. M., Pilewskie, P., and Vukicevic, T.: The Shannon information content of hyperspectral shortwave cloud albedo: Quantification and Practical Applications, J. Geophys. Res., 117, D04025, doi:10.1029/2011JD016771, 2012.

Cornet, C., Buriez, J.-C., Riédi, J., Isaka, H., and Guillemet, B.: Case study of inhomogeneous cloud parameter retrieval from MODIS data, Geophys. Res. Lett., 32, L13807, doi:10.1029/2005GL022791, 2005

Downing, M., Baade, D., Sinclaire, P., Deiries, S., and Christen, F.: CCD riddle: a) signal vs time: linear; b) signal vs variance: non-linear, in: High Energy, Optical, and Infrared Detectors for Astronomy II, 6276, p. 627609, doi:10.1117/12.671457, 2006.

Dury, M. R., Theocharous, T., Harrison, N., Fox, N., and Hilton, M.: Common black coatings - reflectance and ageing characteristics in the $0.32-14.3 \mu \mathrm{m}$ wavelength range, Opt. Commun., 270, 262 272, doi:10.1016/j.optcom.2006.08.038, 2007.

Dworak, A., Charrue, P., Ehm, F., Sliwinski, W., and Sobczak, M.: Middleware Trends And Market Leaders 2011, Proceedings of ICALEPCS2011, CERN-ATS-2011-196, 1334-1337, http://accelconf.web.cern.ch/AccelConf/icalepcs2011/papers/ frbhmult05.pdf (last access: 15 July 2015), 2011.

Dworak, A., Ehm, F., Charrue, P., and Sliwinski, W.: The new CERN Controls Middleware, J. Phys. Conf. Ser., 396, 012017, doi:10.1088/1742-6596/396/1/012017, 2012.

Ehrlich, A., Bierwirth, E., Wendisch, M., Gayet, J.-F., Mioche, G., Lampert, A., and Heintzenberg, J.: Cloud phase identification of Arctic boundary-layer clouds from airborne spectral reflection measurements: test of three approaches, Atmos. Chem. Phys., 8, 7493-7505, doi:10.5194/acp-8-7493-2008, 2008.

Ehrlich, A., Wendisch, M., Bierwirth, E., Gayet, J.-F., Mioche, G., Lampert, A., and Mayer, B.: Evidence of ice crystals at cloud top of Arctic boundary-layer mixed-phase clouds derived from airborne remote sensing, Atmos. Chem. Phys., 9, 9401-9416, doi:10.5194/acp-9-9401-2009, 2009.

Eichler, H., Ehrlich, A., Wendisch, M., Mioche, G., Gayet, J.-F., Wirth, M., Emde, C., and Minikin, A.: Influence of ice crystal shape on retrieval of cirrus optical thickness and effective radius: A case study, J. Geophys. Res.-Atmos., 114, D19203, doi:10.1029/2009JD012215, 2009.

Ewald, F., Zinner, T., and Mayer, B.: Remote sensing of particle size profiles from cloud sides: Observables and retrievals in a $3 \mathrm{D}$ environment, in: AIP Conference Proceedings, 1531, 83-86, AIP Publishing, 2013.

Fischer, J. and Grassl, H.: Detection of Cloud-Top Height from Backscattered Radiances within the Oxygen A Band. Part 1: Theoretical Study, J. Appl. Meteorol., 30, 1245-1259, 1991.

Fossum, E.: CMOS image sensors: electronic camera-on-a-chip, IEEE T. Electron Dev., 44, 1689-1698, doi:10.1109/16.628824, 1997.

Gao, B.-C., Goetz, A. F. H., and Wiscombe, W. J. E.: Cirrus cloud detection from Airborne Imaging Spectrometer data using the $1.38 \mu \mathrm{m}$ water vapor band, Geophys. Res. Lett., 20, 301-304 doi:10.1029/93GL00106, 1993.

Gege, P., Fries, J., Haschberger, P., Schötz, P., Schwarzer, H., Strobl, P., Suhr, B., Ulbrich, G., and Vreeling, W. J.: Calibration facility 
for airborne imaging spectrometers , ISPRS J. Photogramm., 64, 387-397, doi:10.1016/j.isprsjprs.2009.01.006, 2009.

Green, R. O., Eastwood, M. L., Sarture, C. M., Chrien, T. G., Aronsson, M., Chippendale, B. J., Faust, J. A., Pavri, B. E., Chovit, C. J., Solis, M., Olah, M. R., and Williams, O.: Imaging Spectroscopy and the Airborne Visible/Infrared Imaging Spectrometer (AVIRIS), Remote Sens. Environ., 65, 227-248, doi:10.1016/S0034-4257(98)00064-9, 1998.

Hansen, J.: Multiple scattering of polarized light in planetary atmospheres, Part II, Sunlight reflected by terrestrial water clouds, J. Atmos. Sci., 28, 1400-1426, 1971.

Hansen, J. and Pollack, J.: Near-infrared light scattering by terrestrial clouds, J. Atmos. Sci., 27, 265-281, 1970.

Heidinger, A. and Stephens, G.: Molecular line absorption in a scattering atmosphere, Part II: Application to remote sensing in the O2 A-band, J. Atmos. Sci., 57, 1616-1634, 2000.

Hewitt, C., Bishop, P., and Steiger, R.: A Universal Modular ACTOR Formalism for Artificial Intelligence, in: Proceedings of the 3rd International Joint Conference on Artificial Intelligence, IJCAI'73, 235-245, Morgan Kaufmann Publishers Inc., San Francisco, CA, USA, available at: http://dl.acm.org/citation.cfm?id= 1624775.1624804 (last access: 15 July 2015), 1973.

Hintjens, P.: ZeroMQ: The Guide, available at: http://zguide. zeromq.org/page:all (last access: 15 July 2015), 2010.

Holben, B., Eck, T., Slutsker, I., Tanre, D., Buis, J., Setzer, A., Vermote, E., Reagan, J., Kaufman, Y., Nakajima, T. Y., Lavenu, F., Jankowiak, I., and Smirnov, A.: AERONET - a federated instrument network and data archive for aerosol characterization, Remote Sens. Environ., 66, 1-16, 1998.

Houghton, J., Ding, Y., Griggs, D., Noguer, M., van der Linden, P., Dai, X., Maskell, K., and Johnson, C.: Climate change 2001: The scientific basis, Tech. rep., 427-431, 2001.

Hyvarinen, T. S., Herrala, E., and Dall'Ava, A.: Direct sight imaging spectrograph: a unique add-on component brings spectral imaging to industrial applications, in: Digital Solid State Cameras: Designs and Applications, 3302, 165-175, doi:10.1117/12.304581, 1998.

Itten, K. I., Dell'Endice, F., Hueni, A., Kneubühler, M., Schläpfer, D., Odermatt, D., Seidel, F., Huber, S., Schopfer, J., Kellenberger, T., Bühler, Y., D’Odorico, P., Nieke, J., Alberti, E., and Meuleman, K.: APEX - the Hyperspectral ESA Airborne Prism Experiment, Sensors, 8, 6235-6259, 2008.

Jäkel, E., Walter, J., and Wendisch, M.: Thermodynamic phase retrieval of convective clouds: impact of sensor viewing geometry and vertical distribution of cloud properties, Atmos. Meas. Tech., 6, 539-547, doi:10.5194/amt-6-539-2013, 2013.

Janesick, J. R.: Photon Transfer, SPIE Publications, Bellingham, WA, USA, p. 49, 2007.

Jørgensen, R. N.: The VTTVIS line imaging spectrometer: principles, error sources, and calibration, Ris $\emptyset$ National Laboratory, 11-68, 2002.

Kikuchi, N., Nakajima, T., Kumagai, H., Kuroiwa, H., Kamei, A., Nakamura, R., and Nakajima, T. Y.: Cloud optical thickness and effective particle radius derived from transmitted solar radiation measurements: Comparison with cloud radar observations, J. Geophys. Res.-Atmos., 111, D07205, doi:10.1029/2005JD006363, 2006.

Krautstrunk, M. and Giez, A.: The Transition From FALCON to HALO Era Airborne Atmospheric Research, in:
Atmospheric Physics, edited by: Schumann, U., Research Topics in Aerospace, Springer Berlin Heidelberg, 609-624, doi:10.1007/978-3-642-30183-4_37, 2012.

LeBlanc, S. E., Pilewskie, P., Schmidt, K. S., and Coddington, O.: A spectral method for discriminating thermodynamic phase and retrieving cloud optical thickness and effective radius using transmitted solar radiance spectra, Atmos. Meas. Tech., 8, 1361-1383, doi:10.5194/amt-8-1361-2015, 2015.

Lenhard, K., Baumgartner, A., and Schwarzmaier, T.: Independent Laboratory Characterization of NEO HySpex Imaging Spectrometers VNIR-1600 and SWIR-320m-e, IEEE T. Geosci. Remote, 53, 1828-1841, doi:10.1109/TGRS.2014.2349737, 2015.

Marques Vatus, C. and Magnan, P.: Analysis and potentialities of backside-illuminated thinned CMOS imagers, in: Detectors and Associated Signal Processing, 5251, 178-186, doi:10.1117/12.513893, 2004

Marshak, A., Martins, J. V., Zubko, V., and Kaufman, Y. J.: What does reflection from cloud sides tell us about vertical distribution of cloud droplet sizes?, Atmos. Chem. Phys., 6, 5295-5305, doi:10.5194/acp-6-5295-2006, 2006a.

Marshak, A., Platnick, S., Varnai, T., Wen, G., and Cahalan, R. F.: Impact of three-dimensional radiative effects on satellite retrievals of cloud droplet sizes, J. Geophys. Res., 111, D09207, doi:10.1029/2005JD006686, 2006b.

Martins, J. V., Marshak, A., Remer, L. A., Rosenfeld, D., Kaufman, Y. J., Fernandez-Borda, R., Koren, I., Correia, A. L., Zubko, V., and Artaxo, P.: Remote sensing the vertical profile of cloud droplet effective radius, thermodynamic phase, and temperature, Atmos. Chem. Phys., 11, 9485-9501, doi:10.5194/acp-11-94852011, 2011.

Mayer, B., Schröder, M., Preusker, R., and Schüller, L.: Remote sensing of water cloud droplet size distributions using the backscatter glory: a case study, Atmos. Chem. Phys., 4, 12551263, doi:10.5194/acp-4-1255-2004, 2004.

McBride, P. J., Schmidt, K. S., Pilewskie, P., Kittelman, A. S., and Wolfe, D. E.: A spectral method for retrieving cloud optical thickness and effective radius from surface-based transmittance measurements, Atmos. Chem. Phys., 11, 7235-7252, doi:10.5194/acp-11-7235-2011, 2011.

Nakajima, T. Y. and King, M. D.: Determination of the optical thickness and effective particle radius of clouds from reflected solar radiation measurements, Part I: Theory, J. Atmos. Sci, 47, 1878 1893, 1990.

Pilewskie, P., Pommier, J., Bergstrom, R., Gore, W., Rabbette, M., Schmid, B., Hobbs, P. V., and Tsay, S. C.: Solar spectral radiative forcing during the Southern African regional science initiative, J. Geophys. Res.-Atmos., 108, 8486, doi:10.1029/2002JD002411, 2003.

Popp, C., Brunner, D., Damm, A., Van Roozendael, M., Fayt, C., and Buchmann, B.: High-resolution $\mathrm{NO}_{2}$ remote sensing from the Airborne Prism EXperiment (APEX) imaging spectrometer, Atmos. Meas. Tech., 5, 2211-2225, doi:10.5194/amt-5-22112012, 2012.

Rosenfeld, D., Williams, E., Andreae, M. O., Freud, E., Pöschl, U., and Rennó, N. O.: The scientific basis for a satellite mission to retrieve CCN concentrations and their impacts on convective clouds, Atmos. Meas. Tech., 5, 2039-2055, doi:10.5194/amt-52039-2012, 2012. 
Schäfer, M., Bierwirth, E., Ehrlich, A., Heyner, F., and Wendisch, M.: Retrieval of cirrus optical thickness and assessment of ice crystal shape from ground-based imaging spectrometry, Atmos. Meas. Tech., 6, 1855-1868, doi:10.5194/amt-6-1855-2013, 2013.

Schäfer, M., Bierwirth, E., Ehrlich, A., Jäkel, E., and Wendisch, M.: Airborne observations and simulations of three-dimensional radiative interactions between Arctic boundary layer clouds and ice floes, Atmos. Chem. Phys., 15, 8147-8163, doi:10.5194/acp15-8147-2015, 2015.

Schaepman, M., Jehle, M., Hueni, A., D’Odorico, P., Damm, A., Weyermann, J., Schneider, F., Laurent, V., Popp, C., Seidel, F., Lenhard, K., Gege, P., Küchler, C., Brazile, J., Kohler, P., De Vos, L., Meuleman, K., Meynart, R., Schläpfer, D., Kneubühler, M., and Itten, K.: Advanced radiometry measurements and Earth science applications with the Airborne Prism Experiment (APEX), Remote Sens. Environ., 158, 207-219, doi:10.1016/j.rse.2014.11.014, 2015.

Schmidt, K., Pilewskie, P., Platnick, S., Wind, G., Yang, P., and Wendisch, M.: Comparing irradiance fields derived from Moderate Resolution Imaging Spectroradiometer airborne simulator cirrus cloud retrievals with solar spectral flux radiometer measurements, J. Geophys. Res, 112, D24206, doi:10.1029/2007JD008711, 2007.

Schwarzmaier, T., Baumgartner, A., Gege, P., Köhler, C., and Lenhard, K.: DLR's New Traceable Radiance Standard "RASTA", in: International Geoscience and Remote sensing Symposium (IEEE, 2012), 1-4, available at: http://elib.dlr.de/ 78116/ (last access: 15 July 2015), 2012.

Stefanov, K.: A Statistical Model for Signal-Dependent Charge Sharing in Image Sensors, IEEE T. Electron Dev., 61, 110-115, doi:10.1109/TED.2013.2291448, 2014.

Thompson, D. R., Leifer, I., Bovensmann, H., Eastwood, M., Fladeland, M., Frankenberg, C., Gerilowski, K., Green, R. O., Kratwurst, S., Krings, T., Luna, B., and Thorpe, A. K.: Real-time remote detection and measurement for airborne imaging spectroscopy: a case study with methane, Atmos. Meas. Tech., 8, 4383-4397, doi:10.5194/amt-8-4383-2015, 2015.

Twomey, S. and Cocks, T.: Remote sensing of cloud parameters from spectral reflectance in the near-infrared, Beiträge zur Physik der Atmosphäre, 62, 172-179, 1989.

Varnai, T. and Marshak, A.: Observations of three-dimensional radiative effects that influence satellite retrievals of cloud properties, Quarterly Journal of the Hungarian Meteorological Society, 106, 265-278, 2002.
Wendisch, M. and Mayer, B.: Vertical distribution of spectral solar irradiance in the cloudless sky - A case study, Geophys. Res. Lett., 30, 1183, doi:10.1029/2002GL016529, 2003.

Wendisch, M., Müller, D., Schell, D., and Heintzenberg, J.: An airborne spectral albedometer with active horizontal stabilization, J. Atmos. Ocean. Technol., 18, 1856-1866, 2001.

Wendisch, M., Pöschl, U., Andreae, M. O., Machado, L. A. L., Albrecht, R., Schlager, H., Rosenfeld, D., Martin, S. T., Abdelmonem, A., Afchine, A., Araujo, A., Artaxo, P., Aufmhoff, H., Barbosa, H. M. J., Borrmann, S., Braga, R., Buchholz, B., Cecchini, M. A., Costa, A., Curtius, J., Dollner, M., Dorf, M., Dreiling, V., Ebert, V., Ehrlich, A., Ewald, F., Fisch, G., Fix, A., Frank, F., Fütterer, D., Heckl, C., Heidelberg, F., Hueneke, T., Jaekel, E., Jaervinen, E., Jurkat, T., Kanter, S., Kästner, U., Kenntner, M., Kesselmeier, J., Klimach, T., Knecht, M., Kohl, R., Kölling, T., Kraemer, M., Krüger, M., Krisna, T. C., Lavric, J. V., Longo, K., Mahnke, C., Manzi, A. O., Mayer, B., Mertes, S., Minikin, A., Molleker, S., Münch, S., Nillius, B., Pfeilsticker, K., Pöhlker, C., Roiger, A., Rose, D., Rosenow, D., Sauer, D., Schnaiter, M., Schneider, J., Schulz, C., de Souza, R. A. F., Spanu, A., Stock, P., Vila, D., Voigt, C., Walser, A., Walter, D., Weigel, R., Weinzierl, B., Werner, F., Yamasoe, M. A., Ziereis, H., Zinner, T., and Zöger, M.: The ACRIDICON-CHUVA campaign to study tropical deep convective clouds and precipitation using the new German research aircraft HALO, B. Am. Meteorol. Soc., accepted, doi:10.1175/BAMS-D-14-00255.1, 2016.

Wendling, P., Stifter, A., Mayer, B., Fiebig, M., Kiemle, C., Flentje, H., Wendisch, M., Armbruster, W., Leiterer, U., HoyningenHuene, W. V., and Petzold, A.: Aerosol-radiation interaction in the cloudless atmosphere during LACE 98: 2. Aerosol-induced solar irradiance changes determined from airborne pyranometer measurements and calculations, J. Geophys. Res., 107, 8131, doi:10.1029/2000JD000288, 2002.

Zinner, T. and Mayer, B.: Remote sensing of stratocumulus clouds: Uncertainties and biases due to inhomogeneity, J. Geophys. Res., 111, D14209, doi:10.1029/2005JD006955, 2006.

Zinner, T., Marshak, A., Lang, S., Martins, J. V., and Mayer, B.: Remote sensing of cloud sides of deep convection: towards a three-dimensional retrieval of cloud particle size profiles, Atmos. Chem. Phys., 8, 4741-4757, doi:10.5194/acp-8-4741-2008, 2008. 